ESAIM: M2AN 53 (2019) 775-803

https://doi.org/10.1051/m2an/2018056
ESAIM: Mathematical Modelling and Numerical Analysis

www.esaim-m2an.org

\title{
MODELING AND OPTIMIZING A ROAD DE-ICING DEVICE BY A NONLINEAR HEATING
}

\author{
FREDERIC Bernardin ${ }^{1}$ AND ARNAUd MUnCH ${ }^{2 * *}$
}

\begin{abstract}
In order to design a road de-icing device by heating, we consider in the one dimensional setting the optimal control of a parabolic equation with a nonlinear boundary condition of the StefanBoltzmann type. Both the punctual control and the corresponding state are subjected to a unilateral constraint. This control problem models the heating of a road during a winter period to keep the road surface temperature above a given threshold. The one-dimensional modeling used in this work is a first step of the modeling of a road heating device through the circulation of a coolant in a porous layer of the road. We first prove, under realistic physical assumptions, the well-posedness of the direct problem and the optimal control problem. We then perform some numerical experiments using real data obtained from experimental measurements. This model and the corresponding numerical results allow to quantify the minimal energy to be provided to keep the road surface without frost or snow.
\end{abstract}

Mathematics Subject Classification. 49J20, 65C20.

Received March 19, 2018. Accepted September 25, 2018.

\section{INTRODUCTION}

De-icing a road pavement is an important issue in many countries subjected to winter weather conditions that have a strong impact on road maintenance and road safety. The use of salt spreader to ensure the de-icing of pavements can affect the environment close to the road. Also, devices have been implemented to heat the road: electric heating, infrared lamps above the road surface, circulation of a heat transfer fluid in pipes inserted in the road $[10,11,14,20-23]$. The concept of heated pavement is not new. One of the most notable is the Serso project built in Switzerland, with a surface of $1300 \mathrm{~m}^{2}$ : a heat transfer fluid, which draws its energy in the ground, circulates in pipes inserted in the wearing layer pavement [10]. Due to the energy issue being an important concern in all the world, the concept of a positive energy road has emerged since few years. By way of illustration, considering a mean solar energy on the French territory of $1400 \mathrm{kWh} \mathrm{m}^{-2} \mathrm{yr}^{-1}$, the French national road network captures 196 billion $\mathrm{kWhyr}^{-1}$, seven times more energy than needed to ensure for example its surface de-icing by heating.

Under the impulse of the European project R5G (Road of 5th generation), recent research [3,18] has been undertaken on heating the road by circulating a heat transfer fluid within a porous layer of the road. In

\footnotetext{
Keywords and phrases. Optimal control, nonlinear parabolic equation, unilateral constraint, road heating, energy, de-icing.

1 Cerema, Direction Centre-Est, 10 rue Bernard Palissy, 63017 Clermont-Ferrand, France.

2 Laboratoire de Mathématiques Blaise Pascal, Université Clermont Auvergne, UMR CNRS 6620, Campus universitaire des Cézeaux, 3 place Vasarely, 63178 Aubière, France.

*Corresponding author: arnaud.munch@uca.fr
} 

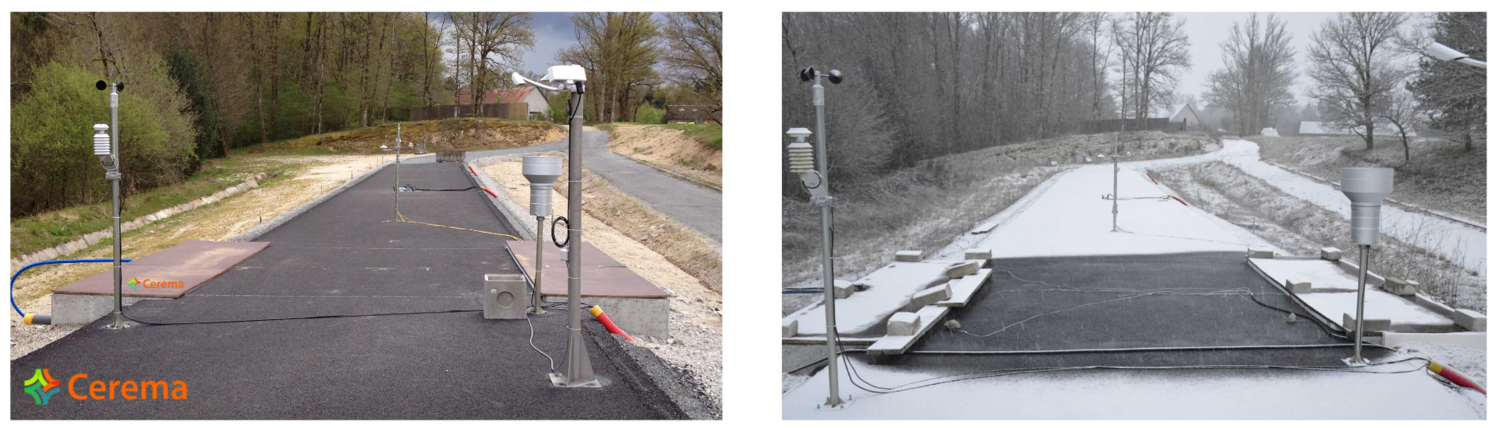

Figure 1. The Egletons demonstrator.

these works, the pavement structure studied is composed of three asphalt layers where the central one is a highly porous draining asphalt through which circulates a fluid (water) via gravitational flow under slant effect. A demonstrator is developed by Cerema (Centre for expertise and engineering on risks, urban and country planning, environment and mobility), EATP (French school of public works) and IFSTTAR (French institute of science and technology for transport, development and networks). This is an experimental roadway constructed in 2014 at Egletons, France. The road with $50 \mathrm{~m}$ in length and $4 \mathrm{~m}$ in width is composed of three layers: a wearing course layer of semi-phaneritic asphalt concrete $0.06 \mathrm{~m}$ thick; a bonding course layer of $0 / 14$ porous asphalt $0.08 \mathrm{~m}$ thick; and a base layer of asphalt concrete with a thickness of $0.05 \mathrm{~m}$. The transversal slope is around $2 \%$ with no longitudinal slope. The demonstrator consists of two parts: a control road and a road in which a fluid (water) circulates through the porous asphalt layer (see Fig. 1). The second one is equipped with two tanks for the supply and recovery of fluid circulating in the bonding course layer. A pump is used to remove fluid from the downstream tank and re-inject it into the upstream tank. Fluid circulation is maintained in the porous asphalt layer by a watertight seal between it and the underlying layer (see Fig. 2). The weather is known on the site thanks to meteorological sensors delivering time-real data on humidity and temperature of the air, radiation fluxes (solar and infrared), wind speed, rainfall and snow. Temperature sensors are inserted in the road from surface until one meter deep in each part of the road (the control one and the one circulated by coolant). To address the energy issue in an experimental way, a heat storage in the granitic soil will be studied by implanting 3 vertical geothermal probes of depth $50 \mathrm{~m}$, allowing to take energy from the ground to heat the

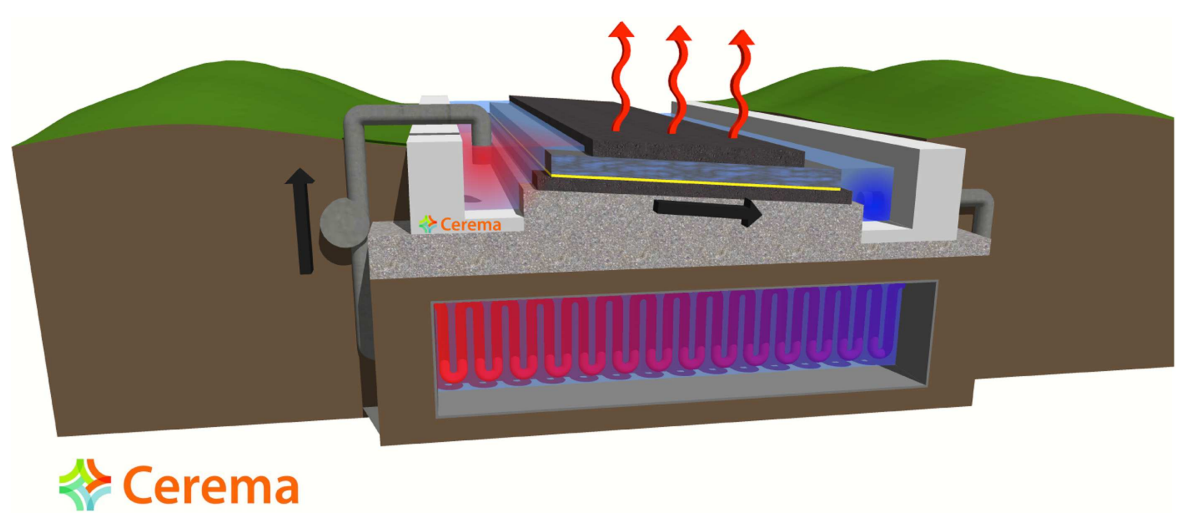

Figure 2. Scheme of the demonstrator (case of heating). 
road in winter, and to store in the ground the heat coming from the road in summer. The demonstrator was presented in a French TV show in 2016 [19].

Whatever the technique used (electrical heating, pipes, porous asphalt), the design of the devices needs to quantify the minimal energy to be provided to keep the road surface without frost or snow, for a given winter meteorological scenario. In order to address this question, we consider in this work the optimal control of a generic heating road model. We are interested in heating during the time interval by a punctual heating source inserted in the road, which is modeled according to its vertical dimension $[2,5,8,12]$. The optimization of the heating through the punctual source leads to an optimal control associated to a one dimensional heat type equation. The underlying functional to minimize represents the energy expended by the positive source over the time period. On a mathematical viewpoint, we then face an optimal control problem, subjected to constraints both on the punctual source and on the temperature state, this latter being solution of a partial differential equation with a nonlinear boundary condition of the Stefan-Boltzmann type.

This paper is organized as follows. In Section 2, we describe respectively the bi-dimensional road heating model and its longitudinal one dimensional reduction leading to the aforementioned nonlinear optimal control problem. Sections 3 and 4 then study the mathematical properties of the one dimensional model and its associated control problem respectively. Section 5 describes the finite dimensional approximation used to solve the control problem then discusses some numerical experiments. Section 6 provides three extensions and perspectives while Section 7 concludes the study.

\section{Modeling of the RoAd heAting AND optimal CONTROL}

We first describe the modeling of the heating thanks to the circulation of a coolant in a bonding porous layer of the road described in Figure 2. Space variables are $x$ along the sub-horizontal transversal axis of the pavement with slant angle $\beta$ and $y$ along the upwards sub-vertical axis, perpendicular to $x$ : we refer to Figure 3 which depicts a transversal two dimensional view of the road. The road is assumed to have no longitudinal slant and to be infinite in its third dimension. $h_{e}$ and $L$ denote the height of the road structure and its length, respectively. The hydraulic regime is assumed stationary with hydraulic parameters independent of temperature $\theta$, expressed in Kelvin. Denoting by $1 \leq i \leq 4$ the subscripts of the road layers, the thermo-hydraulic model is as follows. For $t>0,0 \leq x \leq L$ and $0 \leq y \leq h_{e}$ :

$$
\left\{\begin{array}{l}
C_{i} \frac{\partial \theta}{\partial t}(x, y, t)-\lambda_{i} \Delta \theta(x, y, t)=0, \quad i \in\{1,3,4\} \\
C_{2} \frac{\partial \theta}{\partial t}(x, y, t)+C_{f} v \frac{\partial \theta}{\partial x}(x, y, t)-\left(\lambda_{2}+\phi_{2} \lambda_{f}\right) \Delta \theta(x, y, t)=0 \\
v=-K \frac{H_{2}-H_{1}}{L}
\end{array}\right.
$$

where $C_{i}, \lambda_{i}, \phi_{i}, C_{f}, \lambda_{f}, v$ and $K$ denote specific heat, thermal conductivity and porosity of layer $i$, specific heat and thermal conductivity of the fluid, Darcy fluid velocity along $x$ axis (here supposed to be uniform with respect to $x$ and $y$ ) and hydraulic conductivity of the porous asphalt, respectively. $H_{1}$ and $H_{2}$ represent hydraulic heads imposed upstream and downstream of the fluid circulating in porous draining asphalt layer. The assumption of a saturated fluid circulation corresponds to $H_{1}-H_{2} \geq \beta L$. As mentioned in Figure 3, boundary conditions for problem (2.1) are homogeneous Neumann except for the upstream condition of porous asphalt layer $\left(x=0, e_{1} \leq y \leq e_{1}+e_{2}\right)$ and the road surface condition $(y=0)$. For the first one, the injection temperature of the fluid is imposed:

$$
\forall e_{1} \leq y \leq e_{1}+e_{2}, \forall t \geq 0, \theta(0, y, t)=\theta_{f}(t) .
$$

The second one, that is the road surface boundary condition expresses the energy balance between road and atmosphere

$$
\lambda_{1} \frac{\partial \theta}{\partial y}(x, 0, t)=\sigma \varepsilon(t) \theta^{4}(x, 0, t)+H_{v}(t)\left(\theta(x, 0, t)-\theta_{a}(t)\right)-R_{\mathrm{atm}}(t)-(1-A(t)) R_{g}(t)+L_{f} I(t)
$$




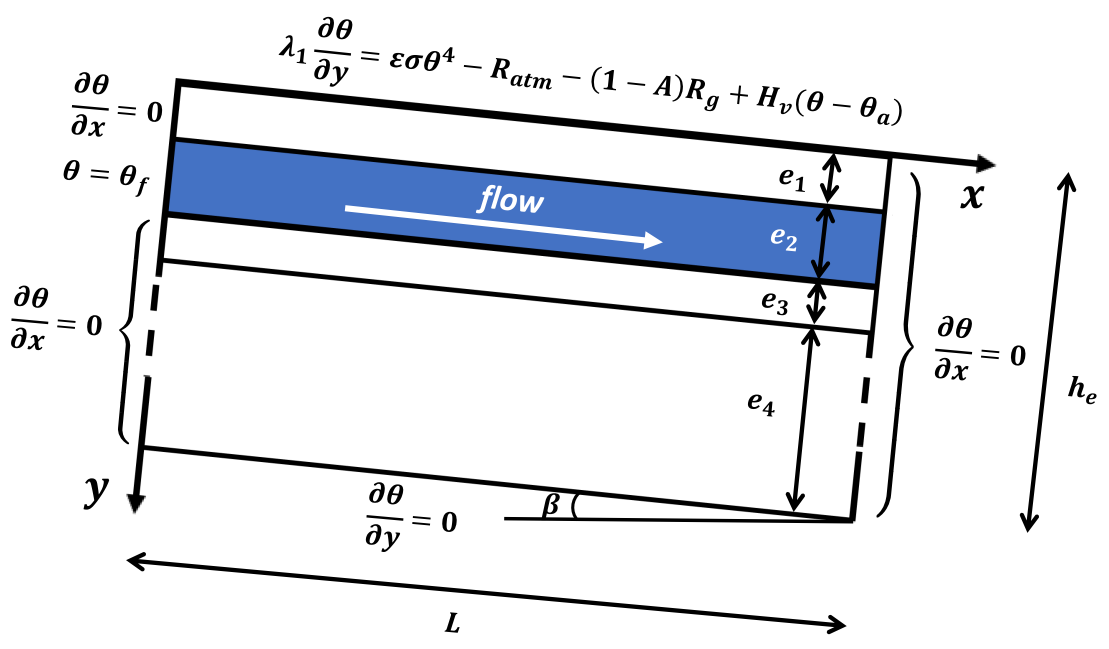

FIgURE 3. Scheme of pavement structure with its limit conditions $\left(\theta_{f}, \theta_{a}\right.$ are the injection temperature of the fluid and the air temperature respectively).

where the following notations are used:

$$
\begin{aligned}
\varepsilon, A & : \text { emissivity and albedo of the road surface, } \\
\sigma & : \text { Stefan-Boltzmann constant }\left(5.67 \times 10^{-8} \mathrm{~W} \mathrm{~m}^{-2} \mathrm{~K}^{-4}\right), \\
R_{\mathrm{atm}}, R_{g} & : \text { atmospheric and global radiation }\left(\mathrm{W} \mathrm{m}^{-2}\right), \\
\theta_{a} & : \text { air temperature }(\mathrm{K}), \\
H_{v} & : \text { convection heat transfer coefficient }\left(\mathrm{W} \mathrm{m}^{-2} \mathrm{~K}\right), \\
I & : \text { snow rate }\left(\mathrm{mm} \mathrm{s}^{-1}\right), \\
L_{f} & : \text { latent heat of fusion of the ice per } \mathrm{kg}\left(\mathrm{J} \mathrm{kg}^{-1}\right) .
\end{aligned}
$$

According to [2], the convection coefficient is defined by $H_{v}=C p_{a} \times \rho_{a}\left(V_{\text {wind }} C_{d}+C_{d_{1}}\right)$ where the following notations are used:

$$
\begin{aligned}
& C p_{a} \text { : thermal capacity }\left(\mathrm{J} \mathrm{kg} \mathrm{K}^{-1}\right) \text { of the air, } \rho_{a}: \text { density of the air }\left(\mathrm{kg} \mathrm{m}^{-3}\right) \text {, } \\
& V_{d}, C_{d_{1}} \text { : two convection coefficients (-). wind velocity }\left(\mathrm{m} \mathrm{s}^{-1}\right),
\end{aligned}
$$

In a first step, we use and study here a one dimensional reduced model obtained by fixing the sub-horizontal axis $x$. The injection temperature term $\theta_{f}$ supported on the boundary $\{0\} \times\left(e_{1}, e_{1}+e_{2}\right)$ is replaced by a punctual heating source $q$ inserted in the road (localized at $y=y_{0} \in\left(0, h_{e}\right)$ ). For any $T>0$, we denote $Q_{T}:=\left(0, h_{e}\right) \times(0, T)$. The evolution of the temperature along the road, now modeled according to its vertical dimension (as explained and used in $[2,5,8]$ ) satisfies the following boundary value system:

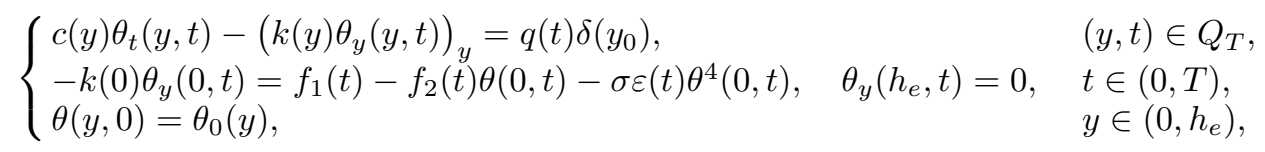

where $\theta=\theta(y, t)$ denotes the temperature in Kelvin at the point $y$ and at time $t$ and where the following notations are used:

$$
\begin{aligned}
& c(y) \text { : volumic heat capacity of the road material at point } y\left(\mathrm{~J} \mathrm{~K}^{-1} \mathrm{~m}^{-3}\right), \\
& k(y) \text { : thermal conductivity of the road material at point } y\left(\mathrm{~W} \mathrm{~K}^{-1} \mathrm{~m}^{-1}\right) .
\end{aligned}
$$


Here and in the sequel, $\theta_{t}$ and $\theta_{y}$ stand for the partial derivative of the function $\theta=\theta(y, t)$ with respect to the space variable $y$ and the time $t$, respectively. The time positive functions $f_{1}$ and $f_{2}$ which appear in the nonlinear boundary conditions at $y=0$ are defined by:

$$
f_{1}(t):=(1-A(t)) R_{g}(t)+R_{\mathrm{atm}}(t)+H_{v}(t) \theta_{a}(t)-L_{f} I(t), \quad f_{2}(t):=H_{v}(t) .
$$

The optimization of the heating through the punctual source $q$ localized at $y=y_{0} \in\left(0, h_{e}\right)$ leads to the following optimal control problem:

$$
\left\{\begin{array}{l}
\inf _{q \in L^{1}(0, T)} J(q):=\int_{0}^{T} q(t) \mathrm{d} t \\
\text { subjected to } \quad q(t) \geq 0, \quad \theta(0, t) \geq \underline{\theta}, \quad \forall t \in(0, T), \quad \theta=\theta(q) \text { solves (2.4) }
\end{array}\right.
$$

for some positive value $\underline{\theta}$, which represents the minimal temperature required. The functional $J$ represents the energy expended by the power $q$ over the period $[0, T]$. Equation $(2.7)$ is thus an optimal control problem subjected to inequality contraints both on the control variable and the temperature state, this latter being solution of a partial differential equation with a nonlinear boundary condition of the Stefan-Boltzmann type. The constraint condition $\theta(0, t) \geq \underline{\theta}$ on the state is not frequent in the optimal control literature as it involves technical developments and is reminiscent of Signorini type conditions (see [9] and also Sect. 6.1). We mention [17] which addresses the controllability of the one dimensional linear heat equation subjected to the boundary condition $\theta_{y}(0, t)=\theta^{4}(0, t)+u(t), u$ being the control. We also mention [1] which addresses the controllability of a linear string submitted to a unilateral obstacle at one extremity.

\section{AnAlysis of the OnE Dimensional MODEL}

Let $H=L^{2}\left(0, h_{e}\right), V=H^{1}\left(0, h_{e}\right)$ and denote by $V^{\prime}$ the dual of $V$. We consider the following boundary value problem: find a solution $\theta$ of

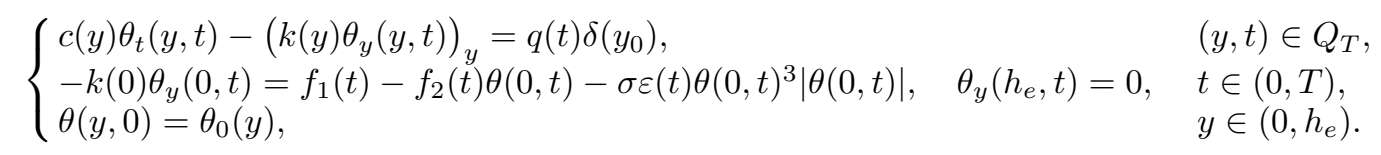

With respect to the system (2.4) introduced in Section 2, the nonlinear term $\sigma \varepsilon(t) \theta^{4}(0, t)$ on the boundary is replaced by the term $\sigma \varepsilon(t) \theta^{3}(0, t)|\theta(0, t)|$, which is monotone w.r.t. $\theta(0, t)$. Actually, we will show in Corollary 3.6 that $\theta \geq 0$.

The weak formulation associated to (3.1) is as follows: find $\theta \in L^{2}(0, T, V)$ such that $\theta_{t} \in L^{2}\left(0, T, V^{\prime}\right)$ and

$$
\begin{aligned}
\left(c \theta_{t}(t), \phi\right)_{H}+a(t, \theta(t), \phi)+\sigma \varepsilon(t) \theta(0, t)^{3}|\theta(0, t)| \phi(0) & \\
& =q(t) \phi\left(y_{0}\right)+f_{1}(t) \phi(0), \quad \forall \phi \in V \text {, a.e.t } \in(0, T)
\end{aligned}
$$

and

$$
\theta(0)=\theta_{0}
$$

$(,)_{H}$ denotes the inner product over $H$ while the form $a: \mathbb{R}^{+} \times V \times V \rightarrow \mathbb{R}$ is defined as follows

$$
\forall(\psi, \phi) \in V^{2}, a(t, \psi, \phi)=\int_{0}^{h_{e}} k(y) \psi_{y} \phi_{y} \mathrm{~d} y+f_{2}(t) \psi(0) \phi(0) .
$$

In order to simplify the notation, we introduce, for almost all $t \in(0, T)$ and $g \in V$, the element $\Phi(g)=$ $g(0)^{3}|g(0)| \delta_{0}$ which can be identified with an element in $V^{\prime}$ so that

$$
\sigma \varepsilon(t)(\Phi(\theta(t)), \phi)_{V^{\prime}, V}=\sigma \varepsilon(t) \theta(0, t)^{3}|\theta(0, t)| \phi(0), \quad t \in(0, T) .
$$

Using that $\|\phi\|:=\left(\left\|\phi_{y}\right\|_{H}^{2}+\phi(0)^{2}\right)^{1 / 2}$ defines a norm equivalent to $\|\phi\|_{V}$, the following holds true. 
Lemma 3.1. Let us assume that there exists $k_{0}>0$ such that $k(y) \geq k_{0}$ for all $y \in\left[0, h_{e}\right]$. The bilinear form a defines a norm equivalent to $\|\cdot\|_{V}$ and

$$
a(t, \psi, \psi) \geq \alpha(t)\|\psi\|_{V}^{2}, \quad a(t, \psi, \phi) \leq \beta(t)\|\psi\|_{V}\|\phi\|_{V} \quad \forall \psi, \phi \in V, \forall t \in(0, T)
$$

with

$$
\alpha(t):=\min \left(k_{0}, f_{2}(t)\right), \quad \beta(t):=\max \left(\|k\|_{L^{\infty}\left(0, h_{e}\right)}, f_{2}(t)\right) .
$$

Let us then introduce the following regularity assumptions:

$$
(\mathcal{H})\left\{\begin{array}{l}
q, f_{1} \in H^{1}(0, T), \theta_{0} \in V,\left(k\left(\theta_{0}\right)_{y}\right)_{y} \in V^{\prime}, \\
c, k \in L^{\infty}\left(0, h_{e}\right), \\
\varepsilon \in H^{1}(0, T), \varepsilon^{-1} \varepsilon_{t} \in L^{\infty}(0, T), \varepsilon(t) \geq 0, \forall t \in(0, T), \\
f_{2} \in H^{1}(0, T), f_{2, t} \in L^{\infty}(0, T) .
\end{array}\right.
$$

Theorem 3.2. Assume the hypothesis $(\mathcal{H})$. Assume moreover that the control $q$ satisfies $q(0)=0$ and that the initial condition $\theta_{0}$ satisfies the compatibility condition

$$
-k(0)\left(\theta_{0}\right)_{y}(0)=f_{1}(0)-f_{2}(0) \theta_{0}(0)-\sigma \varepsilon(0) \theta_{0}^{3}(0)\left|\theta_{0}(0)\right|, \quad\left(\theta_{0}\right)_{y}\left(h_{e}\right)=0,
$$

at the point $y=0$ and $y=h_{e}$, respectively. Then, there exists a unique solution $\theta$ of (3.2) and (3.3) such that

$$
\theta, \theta_{t} \in L^{2}(0, T, V) \cap L^{\infty}(0, T, H) .
$$

Moreover, there exists a constant $C_{1}=C\left(h_{e}, \min _{y \in\left[0, h_{e}\right]} k(y), \min _{t \in[0, T]} f_{2}(t)\right)>0$ such that

$$
\|\sqrt{c} \theta\|_{L^{\infty}(0, T, H)}+\|\theta\|_{L^{2}(0, T, V)} \leq C_{1}\left(\left\|\theta_{0}\right\|_{H}+\|q\|_{L^{2}(0, T)}+\left\|f_{1}\right\|_{L^{2}(0, T)}\right)
$$

and a constant $C_{2}=C\left(C_{1},\left\|f_{2}\right\|_{H^{1}(0, T)},\left\|\varepsilon^{-1} \varepsilon_{t}\right\|_{L^{\infty}(0, T)},\left\|f_{2, t}\right\|_{L^{\infty}(0, T)}\right)>0$ such that

$$
\left\|\sqrt{c} \theta_{t}\right\|_{L^{\infty}(0, T, H)}+\left\|\theta_{t}\right\|_{L^{2}(0, T, V)} \leq C_{2}\left(\left\|k\left(\theta_{0}\right)_{y}\right\|_{H}+\|q\|_{H^{1}(0, T)}+\left\|f_{1}\right\|_{H^{1}(0, T)}\right) .
$$

In particular, this implies that the solution belongs to $C([0, T], V) \subset C\left(\overline{Q_{T}}\right)$.

Proof. The proof follows the arguments developed in [9], Chapter 1, Section 5, based on the monotony of $\Phi$, that is

$$
(\Phi(g), g)_{V^{\prime}, V}=g(0)^{4}|g(0)| \geq 0, \quad \forall g \in V .
$$

The dependence of the emissivity function $\varepsilon$ in (3.2) with respect to the time variable requires however additional developments.

Step 1: Galerkin approximation. We introduce an orthonormal basis $\left\{w_{k}\right\}_{k \geq 1}$ of the separable space $V$ and define, for any integer $m$, by $V_{m}$ the subspace of $V$ generated by $\left\{w_{k}\right\}_{1 \leq k \leq m}$. Moreover, for simplicity, we choose $w_{1}$ in order that $\theta_{0}=w_{1}$. Then, we define the solution $\theta_{m}(t) \in V_{m}$ of the following finite differential system:

$$
\left(c \theta_{m, t}(t), w_{k}\right)_{H}+a\left(t, \theta_{m}(t), w_{k}\right)+\sigma \varepsilon(t)\left(\Phi\left(\theta_{m}(t)\right), w_{k}\right)_{V^{\prime}, V}=q(t) w_{k}\left(y_{0}\right)+f_{1}(t) w_{k}(0), \quad 1 \leq k \leq m
$$

and

$$
\theta_{m}(0)=\theta_{0}
$$

Consequently, the function $\theta_{m}:\left[0, t_{m}\right] \rightarrow V$ is given by $\theta_{m}(t)=\sum_{k=1}^{m} g_{k}(t) w_{k}$ where the time function $\left\{g_{k}\right\}_{1 \leq k \leq m}$ solves a differential system for some time $t_{m}$. The following a priori estimates show that $t_{m}>0$ is independent of $m$. 
Step 2: First a priori estimates. The sum over $k=1, \ldots, m$ of (3.8) first leads to

$$
\left(c \theta_{m, t}(t), \theta_{m}(t)\right)_{H}+a\left(t, \theta_{m}(t), \theta_{m}(t)\right)+\sigma \varepsilon(t)\left(\Phi\left(\theta_{m}(t)\right), \theta_{m}(t)\right)_{V^{\prime}, V}=q(t) \theta_{m}\left(y_{0}, t\right)+f_{1}(t) \theta_{m}(0, t)
$$

leading, in view of (3.7), to

$$
\left.\frac{1}{2} \frac{\mathrm{d}}{\mathrm{d} t}\left\|\sqrt{c} \theta_{m}(t)\right\|_{H}^{2}+a\left(t, \theta_{m}(t), \theta_{m}(t)\right) \leq q(t) \theta_{m}\left(y_{0}\right), t\right)+f_{1}(t) \theta_{m}(0, t)
$$

Lemma 3.1 implies

$$
\frac{1}{2} \frac{\mathrm{d}}{\mathrm{d} t}\left\|\sqrt{c} \theta_{m}(t)\right\|_{H}^{2}+\alpha(t)\left\|\theta_{m}(t)\right\|_{V}^{2} \leq q(t) \theta_{m}\left(y_{0}, t\right)+f_{1}(t) \theta_{m}(0, t) .
$$

Using that $\sup _{y \in\left[0, h_{e}\right]}\left|\theta_{m}(t, y)\right| \leq C_{h_{e}}\left\|\theta_{m}(t)\right\|_{V}$ for some constant $C_{h_{e}}>0$, we get

$$
\frac{1}{2} \frac{\mathrm{d}}{\mathrm{d} t}\left\|\sqrt{c} \theta_{m}(t)\right\|_{H}^{2}+\alpha(t)\left\|\theta_{m}(t)\right\|_{V}^{2} \leq C_{h_{e}}\left(|q(t)|+\left|f_{1}(t)\right|\right)\left\|\theta_{m}(t)\right\|_{V}
$$

The inequality $\left(|q(t)|+\left|f_{1}(t)\right|\right)\left\|\theta_{m}(t)\right\|_{V} \leq \frac{1}{2 \alpha_{2}}\left(|q(t)|+\left|f_{1}(t)\right|\right)^{2}+\frac{\alpha_{2}}{2}\left\|\theta_{m}(t)\right\|_{V}^{2}$ for some $\alpha_{2} \in(0,1)$ leads to

$$
\frac{1}{2} \frac{\mathrm{d}}{\mathrm{d} t}\left\|\sqrt{c} \theta_{m}(t)\right\|_{H}^{2}+\left(\alpha(t)-\frac{C_{h_{e}} \alpha_{2}}{2}\right)\left\|\theta_{m}(t)\right\|_{V}^{2} \leq \frac{C_{h_{e}}}{2 \alpha_{2}}\left(|q(t)|^{2}+\left|f_{1}(t)\right|^{2}\right) .
$$

Taking $\alpha_{2}$ small enough so that $\alpha(t)-\frac{C_{L} \alpha_{2}}{2} \geq 0$, we obtain that

$$
\frac{1}{2}\left\|\sqrt{c} \theta_{m}(t)\right\|_{H}^{2}+\alpha \int_{0}^{t}\left\|\theta_{m}(\tau)\right\|_{V}^{2} \mathrm{~d} \tau \leq \frac{1}{2}\left\|\sqrt{c} \theta_{m}(0)\right\|_{H}^{2}+\frac{C_{h_{e}}}{2 \alpha_{2}} \int_{0}^{t}\left(|q(\tau)|^{2}+\left|f_{1}(\tau)\right|^{2}\right) \mathrm{d} \tau
$$

with $\alpha:=\inf _{t \in(0, T)}\left(\alpha(t)-C_{h_{e}} \frac{\alpha_{2}}{2}\right)$. Finally, we get

$$
\left\|\sqrt{c} \theta_{m}\right\|_{L^{\infty}(0, T, H)}+\left\|\theta_{m}\right\|_{L^{2}(0, T ; V)} \leq C\left(\left\|\theta_{m}(0)\right\|_{H}+\|q\|_{L^{2}(0, T)}+\left\|f_{1}\right\|_{L^{2}(0, T)}\right)
$$

for some positive constant $C=C\left(\min _{y \in\left[0, h_{e}\right]} k(y), \min _{t \in[0, T]} f_{2}(t), \min _{y \in\left[0, h_{e}\right]} c(y), h_{e}\right)$.

Step 3: Second a priori estimates. Taking $t=0$ in (3.8), we obtain

$$
\left(c \theta_{m, t}(\cdot, 0), w_{k}\right)_{H}=-a\left(t, \theta_{m}(\cdot, 0), w_{k}\right)-\sigma \varepsilon(t)\left(\Phi\left(\theta_{m}(\cdot, 0)\right), w_{k}\right)+q(0) w_{k}\left(y_{0}\right)+f_{1}(0) w_{k}, \quad 1 \leq k \leq m .
$$

From (3.6) and the property $q(0)=0,(3.11)$ leads to

$$
\left(c \theta_{m, t}(\cdot, 0), w_{k}\right)_{H}=\left(\left(k(y)\left(\theta_{0}\right)_{y}\right)_{y}, w_{k}\right)_{V^{\prime}, V}, \quad \forall w_{k} \in V,
$$

from which we deduce, following [9], the bound

$$
\left\|c \theta_{m, t}(\cdot, 0)\right\|_{H} \leq\left\|\left(k(y)\left(\theta_{0}\right)_{y}\right)_{y}\right\|_{V^{\prime}} .
$$

Then, by differentiating (3.8) with respect to time, we get, for all $1 \leq k \leq m$

$$
\begin{aligned}
\left(c \theta_{m, t t}(t), w_{k}\right)_{H} & +a\left(t, \theta_{m, t}(t), w_{k}\right)+f_{2, t}(t) \theta_{m}(0, t) w_{k}(0) \\
& +\sigma \frac{\mathrm{d}}{\mathrm{d} t}\left(\varepsilon(t)\left(\Phi\left(\theta_{m}(t)\right), w_{k}\right)\right)=q_{t}(t) w_{k}\left(y_{0}\right)+f_{1, t}(t) w_{k}(0),
\end{aligned}
$$

with

$$
\frac{\mathrm{d}}{\mathrm{d} t}\left(\varepsilon(t)\left(\Phi\left(\theta_{m}(t)\right), w_{k}\right)\right)=\sigma \varepsilon_{t}(t)\left(\theta_{m}(0, t)^{3}\left|\theta_{m}(0, t)\right| w_{k}(0)\right)+\sigma \varepsilon(t)\left(\theta_{m}(0, t)^{3}\left|\theta_{m}(0, t)\right|\right){ }_{t} w_{k}(0) .
$$


Using again (3.7), we have $\left(\theta_{m}(0, t)^{3}\left|\theta_{m}(0, t)\right|\right)_{t} \theta_{m, t}(0, t) \geq 0$ leading to the inequality (recall that $\left.\sigma \varepsilon(t) \geq 0\right)$

$$
\begin{aligned}
\left(c \theta_{m, t t}(t), \theta_{m, t}(t)\right) & +a\left(t, \theta_{m, t}(t), \theta_{m, t}(t)\right)+\sigma \varepsilon_{t}(t) \theta_{m}(0, t)^{3}\left|\theta_{m}(0, t)\right| \theta_{m, t}(0, t) \\
& \leq q_{t}(t) \theta_{m, t}\left(y_{0}, t\right)+f_{1, t}(t) \theta_{m, t}(0, t)-f_{2, t}(t) \theta_{m}(0, t) \theta_{m, t}(0, t)
\end{aligned}
$$

and then to

$$
\begin{aligned}
\frac{1}{2} \frac{\mathrm{d}}{\mathrm{d} t}\left\|\sqrt{c} \theta_{m, t}(t)\right\|_{H}^{2}+\alpha(t)\left\|\theta_{m, t}(t)\right\|_{V}^{2} \leq & -\sigma \varepsilon_{t}(t)\left(\theta_{m}(0, t)^{3}\left|\theta_{m}(0, t)\right| \theta_{m, t}(0, t)\right) \\
& +\left(\left|q_{t}(t)\right|+\left|f_{1, t}(t)\right|\right)\left\|\theta_{m, t}(t)\right\|_{V}+\left|f_{2, t}(t)\right|\left\|\theta_{m}(t)\right\|_{V}\left\|\theta_{m, t}(t)\right\|_{V}
\end{aligned}
$$

with $\alpha$ defined in (3.5). To estimate the remaining boundary term, we multiply (3.8) by $g_{k, t}$ and sum over $k$ leading to the equality

$$
\sigma \varepsilon(t)\left(\Phi\left(\theta_{m}(t)\right), \theta_{m, t}(t)\right)=-\left(c \theta_{m, t}(t), \theta_{m, t}(t)\right)_{H}-a\left(t, \theta_{m}(t), \theta_{m, t}(t)\right)+q(t) \theta_{m, t}\left(y_{0}, t\right)+f_{1}(t) \theta_{m, t}(0, t)
$$

and therefore to

$$
\begin{aligned}
-\sigma \varepsilon_{t}(t) \theta_{m}(0, t)^{3}\left|\theta_{m}(0, t)\right| \theta_{m, t}(0, t)= & -\frac{\varepsilon_{t}(t)}{\varepsilon(t)}\left(\Phi\left(\theta_{m}(t)\right), \theta_{m, t}\right)_{V^{\prime}, V} \\
= & \frac{\varepsilon_{t}(t)}{\varepsilon(t)}\left(\left(c \theta_{m, t}(t), \theta_{m, t}(t)\right)_{H}+a\left(t, \theta_{m}(t), \theta_{m, t}(t)\right)\right. \\
& \left.-q(t) \theta_{m, t}\left(y_{0}, t\right)-f_{1}(t) \theta_{m, t}(0, t)\right) .
\end{aligned}
$$

Using Lemma 3.1, we write

$$
\begin{aligned}
-\sigma \varepsilon_{t}(t) \theta_{m}(0, t)^{3}\left|\theta_{m}(0, t)\right| \theta_{m, t}(0, t) \leq & \left|\frac{\varepsilon_{t}(t)}{\varepsilon(t)}\right|\left(\left\|\sqrt{c} \theta_{m, t}(t)\right\|_{H}^{2}+\beta(t)\left\|\theta_{m}(t)\right\|_{V}\left\|\theta_{m, t}(t)\right\|_{V}\right. \\
& \left.+C_{h_{e}}\left(|q(t)|+\left|f_{1}(t)\right|\right)\left\|\theta_{m, t}(t)\right\|_{V}\right)
\end{aligned}
$$

where $\beta$ is defined in (3.5). Using again several times the Young inequality, we obtain the inequality

$$
\begin{aligned}
\frac{1}{2} \frac{\mathrm{d}}{\mathrm{d} t}\left\|\sqrt{c} \theta_{m, t}(t)\right\|_{H}^{2}+ & \left(\alpha(t)-\frac{\alpha_{1}}{2}-\frac{\alpha_{2}}{2}-\frac{\alpha_{3}}{2}-\frac{\alpha_{4}}{2}\right)\left\|\theta_{m, t}(t)\right\|_{V}^{2} \\
\leq & \left|\frac{\varepsilon_{t}(t)}{\varepsilon(t)}\right|\left\|\sqrt{c} \theta_{m, t}(t)\right\|_{H}^{2}+\frac{1}{2 \alpha_{1}}\left(\left|q_{t}(t)\right|+\left|f_{1, t}(t)\right|\right)^{2}+\frac{1}{2 \alpha_{2}}\left|f_{2, t}(t)\right|^{2}\left\|\theta_{m}(t)\right\|_{V}^{2} \\
& +\frac{1}{2 \alpha_{3}}\left|\frac{\varepsilon_{t}(t)}{\varepsilon(t)}\right|^{2} \beta^{2}(t)\left\|\theta_{m}(t)\right\|_{V}^{2}+\frac{1}{2 \alpha_{4}}\left|\frac{\varepsilon_{t}(t)}{\varepsilon(t)}\right|^{2}\left(|q(t)|+\left|f_{1}(t)\right|\right)^{2}
\end{aligned}
$$

for any $\alpha_{1}, \alpha_{2}, \alpha_{3}, \alpha_{4}>0$ small enough, from which we deduce that

$$
\begin{aligned}
\frac{1}{2}\left\|\sqrt{c} \theta_{m, t}(t)\right\|_{H}^{2}+\alpha \int_{0}^{t}\left\|\theta_{m, t}(\tau)\right\|_{V}^{2} \mathrm{~d} \tau \leq & \frac{1}{2}\left\|\sqrt{c} \theta_{m, t}(0)\right\|_{H}^{2}+\int_{0}^{t}\left|\frac{\varepsilon_{t}(\tau)}{\varepsilon(\tau)}\right|\left\|\sqrt{c} \theta_{m, t}(\tau)\right\|_{H}^{2} \mathrm{~d} \tau \\
& +\alpha_{5}\left\|\theta_{m}\right\|_{L^{2}(0, T, V)}^{2}+\alpha_{6}
\end{aligned}
$$

with

$$
\begin{aligned}
& \alpha:=\inf _{t \in(0, T)}\left(\alpha(t)-\frac{1}{2}\left(\alpha_{1}+\alpha_{2}+\alpha_{3}+\alpha_{4}\right)\right), \quad \alpha_{5}:=\frac{\left\|f_{2, t}\right\|_{L^{\infty}(0, T)}^{2}}{2 \alpha_{2}}+\frac{1}{2 \alpha_{3}}\left\|\frac{\varepsilon_{t}}{\varepsilon} \beta\right\|_{L^{\infty}(0, T)}^{2}, \\
& \alpha_{6}:=\frac{1}{2 \alpha_{1}}\left(\left\|q_{t}\right\|_{L^{2}(0, T)}^{2}+\left\|f_{1, t}\right\|_{L^{2}(0, T)}^{2}\right)+\frac{1}{2 \alpha_{4}}\left\|\frac{\varepsilon_{t}}{\varepsilon}\right\|_{L^{\infty}(0, T)}^{2}\left(\|q\|_{L^{2}(0, T)}^{2}+\left\|f_{1}\right\|_{L^{2}(0, T)}^{2}\right) .
\end{aligned}
$$


A Gronwall type lemma allows to deduce that, for all $t \in(0, T)$,

$$
\left\|\sqrt{c} \theta_{m, t}(t)\right\|_{H}^{2} \leq\left(\left\|\sqrt{c} \theta_{m, t}(0)\right\|_{H}^{2}+\alpha_{5}\left\|\theta_{m}\right\|_{L^{2}(0, T, V)}^{2}+\alpha_{6}\right)\left(1+2\left\|\varepsilon^{-1} \varepsilon_{t}\right\|_{L^{\infty}(0, T)} e^{t\left\|\varepsilon^{-1} \varepsilon_{t}\right\|_{L^{\infty}(0, T)}}\right) .
$$

Uniform estimates (3.10) and (3.12) then imply that $\sqrt{c} \theta_{m, t}$ is uniformly bounded in $L^{\infty}(0, T, H)$ with respect to the parameter $m$. Inequality (3.14) then implies that $\theta_{m, t}$ is uniformly bounded in $L^{2}(0, T, V)$ as well, and that there exists a constant $C>0$ such that

$$
\left\|\sqrt{c} \theta_{m, t}\right\|_{L^{\infty}(0, T, H)}+\left\|\theta_{m, t}\right\|_{L^{2}(0, T, V)} \leq C\left(\left\|\left(k(y)\left(\theta_{0}\right)_{y}\right)_{y}\right\|_{V^{\prime}}+\|q\|_{H^{1}(0, T)}+\left\|f_{1}\right\|_{H^{1}(0, T)}\right) .
$$

Step 4: Limit as $m \rightarrow \infty$. From (3.12) and (3.15), $\theta_{m}$ is bounded uniformly in $L^{\infty}(0, T, V)$ and therefore, from (3.8), $\Phi\left(\theta_{m}\right)$ is bounded in $L^{\infty}\left(0, T, V^{\prime}\right)$. We can extract a subsequence $\left\{\theta_{\mu}\right\}_{\mu>0}$ such that, as $\mu \rightarrow \infty$,

$$
\begin{aligned}
\theta_{\mu} \rightarrow \theta & \text { weakly star in } L^{\infty}(0, T, V), \\
\theta_{\mu, t} \rightarrow \theta_{t} & \text { weakly star in } L^{2}(0, T, V) \text { and weakly star in } L^{\infty}(0, T, H), \\
\Phi^{\prime}\left(\theta_{\mu}\right) \rightarrow Q & \text { weakly star in } L^{\infty}\left(0, T, V^{\prime}\right),
\end{aligned}
$$

and

$$
\|\theta\|_{L^{\infty}(0, T, V)}+\left\|\theta_{t}\right\|_{L^{\infty}(0, T, V)}+\left\|\theta_{t}\right\|_{L^{\infty}(0, T, H)} \leq C .
$$

The previous convergences imply notably that $\theta_{\mu}(0) \rightarrow \theta_{0}$ as $\mu \rightarrow \infty$ in $V$ separable so that $\theta_{0}=\theta(0)$.

Writing (3.8) for the subsequence $\theta_{\mu}$ and using (3.16), we obtain that $\theta$ and $Q$ satisfy the equality

$$
\left(c \theta_{t}(t), w_{k}\right)_{H}+a\left(t, \theta(t), w_{k}\right)+\left(Q, w_{k}\right)_{V^{\prime}, V}=q(t) w_{k}\left(y_{0}\right)+f_{1}(t) w_{k}(0), \quad 1 \leq k \leq m
$$

leading to (using that $V_{m}$ is dense in $V$ )

$$
\left(\theta_{t}(t), v\right)_{H}+a(t, \theta(t), v)+(Q, v)_{V^{\prime}, V}=q(t) v\left(y_{0}\right)+f_{1}(t) v(0), \quad \forall v \in V .
$$

It remains to pass to the limit in the nonlinear term in order to show the equality $Q=\Phi(\theta)$ in $L^{\infty}\left(0, T, V^{\prime}\right)$. To do that, we use the equality (3.8): precisely, let $\phi \in L^{2}(0, T, V)$ and let

$$
U_{\mu}:=\int_{0}^{T}\left(\Phi\left(\theta_{\mu}\right)-\Phi(\phi), \theta_{\mu}-\phi\right)_{V^{\prime}, V} \mathrm{~d} t
$$

so that from (3.8),

$$
\begin{aligned}
U_{\mu}= & -\int_{0}^{T}\left(\left(c \theta_{\mu}^{\prime}(t), \theta_{\mu}(t)\right)_{H}+a\left(t, \theta_{\mu}(t), \theta_{\mu}(t)\right)-q(t) \theta_{\mu}\left(y_{0}, t\right)-f_{1}(t) \theta_{\mu}(0, t)\right) \mathrm{d} t \\
& -\int_{0}^{T}\left(\Phi\left(\theta_{\mu}\right), \phi\right) d t-\int_{0}^{T}\left(\Phi(\phi), \theta_{\mu}-\phi\right) \mathrm{d} t .
\end{aligned}
$$

The monotony of $\Phi$ implies that $U_{\mu} \geq 0$. Moreover, the equality $-\int_{0}^{T}\left(c \theta_{\mu}^{\prime}(t), \theta_{\mu}(t)\right)_{H} \mathrm{~d} t=-\frac{1}{2}\left\|\sqrt{c} \theta_{\mu}(T)\right\|_{H}^{2}+$ $\frac{1}{2}\left\|\sqrt{c} \theta_{0}\right\|_{H}^{2}$ and the weak star convergence of $\theta_{\mu}$ in $L^{\infty}(0, T, V)$ (and so in $L^{\infty}(0, T, H)$ ) allows to take the lim sup of $U_{\mu}$. Precisely, from the upper semi-continuity of $v \rightarrow-\|v\|_{H}^{2}$, we write that $\lim \sup \left(-\left\|\sqrt{c} \theta_{\mu}(t)\right\|_{H}^{2}\right) \leq$ $-\|\sqrt{c} \theta(t)\|_{H}^{2}$ for all $t \in(0, T)$ and therefore,

$$
\begin{aligned}
0 \leq & \lim \sup U_{\mu} \leq-\frac{1}{2}\|\sqrt{c} \theta(T)\|_{H}^{2}+\frac{1}{2}\left\|\sqrt{c} \theta_{0}\right\|_{H}^{2} \\
& -\int_{0}^{T}\left(a(t, \theta(t), \theta(t))-q(t) \theta\left(y_{0}, t\right)-f_{1}(t) \theta(0, t)\right) \mathrm{d} t-\int_{0}^{T}(Q, \phi) \mathrm{d} t-\int_{0}^{T}\left(\Phi^{\prime}(\phi), \theta-\phi\right) \mathrm{d} t .
\end{aligned}
$$


On the other hand, the integration over $(0, T)$ of $(3.17)$ with $v=\theta(t) \in V$ leads to

$$
-\frac{1}{2}\|\sqrt{c} \theta(T)\|_{H}^{2}+\frac{1}{2}\left\|\sqrt{c} \theta_{0}\right\|_{H}^{2}-\int_{0}^{T}\left(a(t, \theta(t), \theta(t))-q(t) \theta\left(y_{0}, t\right)-f_{1}(t) \theta(0, t)\right) \mathrm{d} t=\int_{0}^{T}(Q, \theta(t))_{V^{\prime}, V} \mathrm{~d} t .
$$

The sum of the two previous relations implies that $\int_{0}^{T}(Q-\Phi(\theta), \theta-\phi) \mathrm{d} t \geq 0$ for all $\phi$; in particular, for $\phi=\theta-\lambda \psi, \lambda \in \mathbb{R}, \theta \in L^{2}(0, T, V)$, the previous inequality gives $\int_{0}^{T}(Q-\Phi(\theta-\lambda \psi), \lambda \psi) \mathrm{d} t \geq 0$ and then by making $\lambda \rightarrow 0^{+}$and $\lambda \rightarrow 0^{-}$

$$
\int_{0}^{T}(Q-\Phi(\theta), \psi)_{V^{\prime}, V} \mathrm{~d} t=0 \quad \forall \psi \in L^{2}(0, T, V)
$$

which allows to write $Q=\Phi(\theta)$ in $L^{\infty}\left(0, T, V^{\prime}\right)$.

Step 5: Uniqueness. By contradiction, let $\theta, \theta^{\star}$ be two distinct solutions of (3.2) and let $w=\theta-\theta^{\star}$. Taking $\phi=w(t) \in V$ in $(3.2)$, we obtain

$$
\left(c w_{t}(t), w(t)\right)_{H}+a(t, w(t), w(t))=-\left(\Phi(\theta(t))-\Phi\left(\theta^{\star}(t)\right), w\right)_{H} \leq 0 \text {, a.e.t } \in(0, T)
$$

by the monotony of $\Phi$. Since $c>0$, we deduce that $\left(c w^{\prime}(t), w(t)\right)_{H} \leq 0$ then $\|\sqrt{c} w(t)\|_{H} \leq \|$ $\sqrt{c} w(0) \|_{H}=0$.

Remark 3.3. The arguments of step 5 allow to show that if $\theta_{1}$ and $\theta_{2}$ are solution of (3.2) and (3.3) associated to $q_{1}$ and $q_{2}$, respectively, the other data being equals, then there exists a constant $C>0$ such that

$$
\left\|\theta_{1}-\theta_{2}\right\|_{L^{\infty}(0, t, H)}+\left\|\theta_{1}-\theta_{2}\right\|_{L^{2}(0, t, V)} \leq C\left\|q_{1}-q_{2}\right\|_{L^{2}(0, t)}, \quad \forall t>0 .
$$

Remark 3.4. A similar well-posedness result holds true if the Neumann boundary condition at $y=h_{e}$ is replaced by a non homogenous Dirichlet boundary $\theta\left(h_{e}, \cdot\right)=\theta_{d} \in \mathbb{R}$. This latter, which is physically relevant if the height $h_{e}$ is large enough, will be used in the numerical Section 5 .

Moreover, the solution enjoys the following comparison principle.

Proposition 3.5. Assume the hypothesis of Theorem 3.2. Let $\theta$ and $\hat{\theta}$ the solutions of (3.2) and (3.3) associated to the pair $\left(q, \theta_{0}\right)$ and $\left(\hat{q}, \hat{\theta}_{0}\right)$, respectively. If $q \geq \hat{q}$ in $[0, T]$ and $\theta_{0} \geq \hat{\theta}_{0}$ in $[0, L]$, then $\theta \geq \hat{\theta}$ in $Q_{T}$.

Proof. Let $m=\hat{\theta}-\theta \in V$ and $m^{+}=\max (0, m)$ the positive part of $m$. Taking $\phi=m^{+}(t) \in V$ in (3.2), we obtain

$$
-\left(c m_{t}(t), m^{+}(t)\right)_{H}-a\left(t, m(t), m^{+}(t)\right)+\left(\Phi(\theta(t))-\Phi(\hat{\theta}(t)), m^{+}\right)_{V^{\prime}, V}=(q(t)-\hat{q}(t)) m^{+}\left(y_{0}\right) \text {, a.e.t } \in(0, T) .
$$

The right hand side is positive, while, in view of the monotony of $\Phi$, the term $\left(\Phi(\theta(t))-\Phi(\hat{\theta}(t)), m^{+}\right)_{V^{\prime}, V}$ is negative. Therefore,

$$
\left(c m_{t}(t), m^{+}(t)\right)_{H}+a\left(t, m(t), m^{+}(t)\right) \leq 0 \text {, a.e.t } \in(0, T)
$$

and using that $a\left(t, m(t), m^{+}(t)\right)=a\left(t, m^{+}(t), m^{+}(t)\right)$ and that $\left(c m_{t}(t), m^{+}(t)\right)_{H}=\left(c\left(m^{+}(t)\right)_{t}, m^{+}(t)\right)_{H}$, we have

$$
\left(c\left(m^{+}\right)_{t}(t), m^{+}(t)\right)_{H}+a\left(t, m^{+}(t), m^{+}(t)\right) \leq 0 \text {, a.e.t } \in(0, T) .
$$

leading to $\frac{\mathrm{d}}{\mathrm{d} t}\left\|\sqrt{c} m^{+}(t)\right\|_{H}^{2} \leq 0$ and $\left\|\sqrt{c} m^{+}(t)\right\|_{H}^{2} \leq\left\|\sqrt{c} m^{+}(0)\right\|_{H}^{2}=0$ since $m(0)=\hat{\theta}(0)-\theta(0) \leq 0$.

In particular, since the time function $f_{1}$ is nonnegative, we deduce the following property.

Corollary 3.6. Assume the hypothesis of Theorem 3.2. Let $\theta$ the solution of (3.2) and (3.3) associated to the pair $\left(q, \theta_{0}\right)$. If $q \geq 0$ in $[0, T]$ and $\theta_{0} \geq 0$ in $\left[0, h_{e}\right]$, then $\theta \geq 0$ in $\overline{Q_{T}}$.

It results that if the source term and if the initial condition are nonnegative, then the boundary value problem (3.1) coincides with the initial one (2.4). 


\section{Analysis of the optimal control problem}

\subsection{Well-posedness of the optimal control problem}

Let $\alpha>0$ and $\underline{\theta}>0$. We assume that the initial condition $\theta_{0}$ is nonnegative a.e. in $\left[0, h_{e}\right]$. In view of the regularity assumption on $q$ in Theorem 3.2, we introduce the following Tychonov regularization of the optimal control problem (2.7):

$$
\inf _{(\theta, q) \in \mathcal{C}} J_{\alpha}(\theta, q):=\frac{1}{2}\left(\|q\|_{L^{1}(0, T)}^{2}+\alpha\|q\|_{H^{1}(0, T)}^{2}\right)
$$

where the constraint set is given by

$$
\mathcal{C}:=\left\{q \in H^{1}(0, T), q(0)=0, q(t) \geq 0, \theta(0, t) \geq \underline{\theta}, \forall t \in[0, T], \theta=\theta(q) \text { solves }(2.4)\right\} .
$$

Consequently, if the control function $q$ belongs to $\mathcal{C}$, then from Corollary 3.6, the weak formulation of $(2.4)$ coincides with the weak formulation of (3.1) and is well-posed. We relax the second inequality constraint from $\mathcal{C}$ and introduce the equivalent extremal problem:

$$
\left(\mathcal{P}_{\psi}\right): \quad \inf _{q \in \mathcal{D}} J_{\alpha}(q):=\frac{1}{2}\left(\|q\|_{L^{1}(0, T)}^{2}+\alpha\|q\|_{H^{1}(0, T)}^{2}\right)+\psi_{K}(q)
$$

where $\psi_{K}$ is the indicator function of $K$, that is $\psi_{K}(q)=0$ if $q \in K$ and $\psi_{K}(q)=+\infty$ else with $K=\{q \in$ $H^{1}(0, T)$ s.t. $\int_{0}^{T}\left((\theta(0, t)-\underline{\theta})^{-}\right)^{2} \mathrm{~d} t=0$ where $\theta=\theta(q)$ solves $\left.(2.4)\right\}$ and

$$
\mathcal{D}=\left\{q \in H^{1}(0, T), q(0)=0, q(t) \geq 0, \quad \forall t \in(0, T)\right\} .
$$

Lemma 4.1. Let us assume that $\theta_{0} \geq \underline{\theta}$ on $\left(0, h_{e}\right)$. If one of the following hypotheses holds true,

$$
\left(\mathcal{H}_{1}\right) \quad y_{0}=0 ; \quad\left(\mathcal{H}_{2}\right) \quad f_{1}(t)-f_{2}(t) \underline{\theta}-\sigma \varepsilon(t) \underline{\theta}^{4} \geq 0, \quad \forall t \in(0, T),
$$

then $K$ is not empty.

Proof. Let $Z:=\theta-\underline{\theta}$ where $\theta$ solves $(3.2)$ and let $Z^{-}=\min (0, Z)$ be the negative part of $Z$. We have for a.e. $t \in(0, T)$,

$$
\left(c \theta_{t}(t), Z^{-}(t)\right)_{H}+a\left(t, \theta(t), Z^{-}(t)\right)+\left(\Phi(\theta(t)), Z^{-}(t)\right)_{V^{\prime}, V}=q(t) Z^{-}\left(y_{0}, t\right)+f_{1}(t) Z^{-}(0, t),
$$

equivalently,

$$
\begin{aligned}
\left(c Z_{t}^{-}(t), Z^{-}(t)\right)_{H}+a\left(t, Z^{-}(t), Z^{-}(t)\right)= & -\sigma \varepsilon(t) \theta(0, t)^{4} Z^{-}(0, t) \\
& +q(t) Z^{-}\left(y_{0}, t\right)+\left(f_{1}(t)-f_{2}(t) \underline{\theta}\right) Z^{-}(0, t),
\end{aligned}
$$

a.e. in $(0, T)$. We now claim that

$$
-\sigma \varepsilon(t) \theta(0, t)^{4} Z^{-}(0, t) \leq-\sigma \varepsilon(t) \underline{\theta}^{4} Z^{-}(0, t), \quad \text { a.e. } t \in(0, T) .
$$

This is clear if $Z^{-}(0, t)=0$. If $Z^{-}(0, t)<0$, then $0<\theta(0, t)<\underline{\theta}$ and then $\theta(0, t)^{4}<\underline{\theta}^{4}$ leading to the result since $-\sigma \varepsilon(t) Z^{-}(0, t)>0$. Consequently, a.e. in $(0, T)$,

$$
\begin{aligned}
\left(c Z_{t}^{-}(t), Z^{-}(t)\right)_{H} & +a\left(t, Z^{-}(t), Z^{-}(t)\right) \leq q(t) Z^{-}\left(y_{0}, t\right)+\left(f_{1}(t)-f_{2}(t) \underline{\theta}-\sigma \varepsilon(t) \underline{\theta}^{4}\right) Z^{-}(0, t) \\
& \leq q(t)\left(Z^{-}\left(y_{0}, t\right)-Z^{-}(0, t)\right)+\left(q(t)+f_{1}(t)-f_{2}(t) \underline{\theta}-\sigma \varepsilon(t) \underline{\theta}^{4}\right) Z^{-}(0, t) .
\end{aligned}
$$


If hypothesis $\left(\mathcal{H}_{2}\right)$ holds true, the right hand side is negative and we conclude that $\left\|\sqrt{c} Z^{-}(t)\right\|_{H} \leq$ $\left\|\sqrt{c} Z^{-}(0)\right\|_{H}=0$ for all $t \geq 0$, since $q \geq 0$. Remark in particular that $\left(\mathcal{H}_{2}\right)$ holds true if $\underline{\theta} \leq 0$ and we re-obtain the maximum principle, Corollary 3.6. On the other hand, if $y_{0}=0$, we get the same conclusion as soon as $q(t)+f_{1}(t)-f_{2}(t) \underline{\theta}-\sigma \varepsilon(t) \underline{\theta}^{4} \geq 0$ for all $t$, that is, if $q$ is large enough.

According to the physical intuition, if the heat source is located on the surface, then the temperature can be maintained as large as requested. In view of the continuity of the temperature $\theta(y, t)$ with respect to the longitudinal axis $y$, the same result holds true if $y_{0}$ is negative but closed enough to 0 . Actually, we believe that the same result holds true if the heat source, located on any $y_{0}<0$, is large enough, the temperature $\theta$ being a continuous and monotonous function of $q$, see Proposition 3.5.

Lemma 4.2. $K$ is a closed convex subset of $H^{1}(0, T)$ and so $\psi_{K}$ is convex and lower semi-continuous.

Proof. Let $q, \bar{q} \in K$ and check that for any $\lambda \in(0,1), \lambda q+(1-\lambda) \bar{q}$ belongs to $K . q \in K$ implies that for all $t \in[0, T], \theta_{q}(t) \geq \underline{\theta}$ where $\theta_{q}$ solves $(2.4)$ associated to the source $q$. Similarly, $\theta_{\bar{q}}(t) \geq \underline{\theta}$. Let $\theta_{\lambda}$ be the solution associated to the control function $\lambda q+(1-\lambda) \bar{q}$ and let $[\theta]:=\lambda \theta_{q}+(1-\lambda) \theta_{\bar{q}}$. From (3.2), $\theta_{\lambda}$ and $[\theta]$, respectively solves

$$
\begin{aligned}
\left(\theta_{\lambda, t}(t), \phi\right)_{H} & +a\left(t, \theta_{\lambda}(t), \phi\right)+\left(\Phi\left(\theta_{\lambda}(t)\right), \phi\right)_{V^{\prime}, V} \\
& =(\lambda q(t)+(1-\lambda) \bar{q}(t)) \phi\left(y_{0}\right)+f_{1}(t) \phi(0), \quad \forall \phi \in V \text {, a.e.t } \in(0, T)
\end{aligned}
$$

and

$$
\begin{aligned}
\left([\theta]_{t}(t), \phi\right)_{H} & +a(t,[\theta](t), \phi)+\left(\lambda \Phi\left(\theta_{q}(t)\right)+(1-\lambda) \Phi\left(\theta_{\bar{q}}(t)\right), \phi\right)_{V^{\prime}, V} \\
& =(\lambda q(t)+(1-\lambda) \bar{q}(t)) \phi\left(y_{0}\right)+f_{1}(t) \phi(0), \quad \forall \phi \in V \text {, a.e. } t \in(0, T) .
\end{aligned}
$$

The difference $W:=\theta_{\lambda}-[\theta]$ solves

$$
\left(W_{t}(t), \phi\right)_{H}+a(t, W, \phi)+\left(\Phi\left(\theta_{\lambda}(t)\right)-\lambda \Phi\left(\theta_{q}(t)\right)-(1-\lambda) \Phi\left(\theta_{\bar{q}}(t)\right), \phi\right)_{V^{\prime}, V}=0, \forall \phi \in V \text {, a.e.t } \in(0, T) .
$$

Taking $\phi=W(t)$, integrating over $(0, t)$ and using that $W(0)=0$, we arrive at

$$
\begin{aligned}
\frac{1}{2}\|W(t)\|_{H}^{2} & +\int_{0}^{t} a(s, W(s), W(s)) \mathrm{d} s \\
& +\int_{0}^{t}\left(\Phi\left(\theta_{\lambda}(s)\right)-\lambda \Phi\left(\theta_{q}(s)\right)-(1-\lambda) \Phi\left(\theta_{\bar{q}}(s)\right), \phi\right)_{V^{\prime}, V} \mathrm{~d} s=0, \quad \forall t \geq 0
\end{aligned}
$$

leading to

$$
\begin{aligned}
\frac{1}{2}\|W(t)\|_{H}^{2} & +\int_{0}^{t}\left(\Phi\left(\theta_{\lambda}(s)\right)-\lambda \Phi\left(\theta_{q}(s)\right)-(1-\lambda) \Phi\left(\theta_{\bar{q}}(s)\right), \phi\right)_{V^{\prime}, V} \mathrm{~d} s \\
& =-\int_{0}^{t} a(s, W(s), W(s)) \mathrm{d} s, \forall \lambda \in(0,1), \forall t>0 .
\end{aligned}
$$

If $\int_{0}^{t^{\star}} a(s, W(s), W(s)) \mathrm{d} s=0$ for some $t^{\star}>0$, then $W(s)=0$ for all $s \in\left[0, t^{\star}\right]$ and then $[\theta](0, s)=$ $\lambda \theta_{q}(0, s)+(1-\lambda) \theta_{\bar{q}}(0, s) \geq \underline{\theta}$ for all $s \in\left[0, t^{\star}\right]$. The discussion is then reduced to the interval $\left(t^{\star}, T\right)$. Without loss of generality, let us then assume that $\int_{0}^{t} a(s, W(s), W(s)) \mathrm{d} s>0$ for all $t>0$ so that

$$
\int_{0}^{t}\left(\Phi\left(\theta_{\lambda}(s)\right)-\lambda \Phi\left(\theta_{q}(s)\right)-(1-\lambda) \Phi\left(\theta_{\bar{q}}(s)\right), W(s)\right)_{V^{\prime}, V} \mathrm{~d} s<0, \quad \forall t>0 .
$$


Suppose now that for all $s \in(0, t), W(0, s)=\theta_{\lambda}(0, s)-[\theta](0, s) \leq 0$. The convexity of $x \rightarrow x^{4}$ leads to $[\theta]^{4}(0, s) \leq \lambda \theta_{q}^{4}(0, s)+(1-\lambda) \theta_{\bar{q}}^{4}(0, s)$, then to

$$
\theta_{\lambda}^{4}(0, s)-[\theta]^{4}(0, s) \geq \theta_{\lambda}^{4}(0, s)-\lambda \theta_{q}^{4}(0, s)-(1-\lambda) \theta_{\bar{q}}^{4}(0, s)
$$

and eventually to

$$
W(0, s)\left(\theta_{\lambda}^{4}(0, s)-\lambda \theta_{q}^{4}(0, s)-(1-\lambda) \theta_{\bar{q}}^{4}(0, s)\right) \geq W(0, s)\left(\theta_{\lambda}^{4}(0, s)-[\theta]^{4}(0, s)\right) .
$$

But, the right-hand side is exactly $(\alpha-\beta)\left(\alpha^{4}-\beta^{4}\right)=(\alpha-\beta)^{2}\left(\alpha^{2}+\beta^{2}\right)(\alpha+\beta)$ with

$$
\alpha=\theta_{\lambda}(0, s) \geq \underline{\theta}>0, \quad \beta=\lambda \theta_{q}(0, s)+(1-\lambda) \theta_{\bar{q}}(0, s) \geq \underline{\theta}>0
$$

and is therefore positive since $\beta=[\theta](0, s) \geq \underline{\theta}$ by assumption and since $\alpha=\theta_{\lambda}(0, s) \geq 0$ (in view of the maximum principle). Consequently,

$$
\begin{aligned}
\int_{0}^{t}\left(\Phi\left(\theta_{\lambda}(s)\right)\right. & \left.-\lambda \Phi\left(\theta_{q}(s)\right)-(1-\lambda) \Phi\left(\theta_{\bar{q}}(s)\right), W(s)\right)_{V^{\prime}, V} \mathrm{~d} s \\
& =\int_{0}^{t} \sigma \varepsilon(t) W(0, s)\left(\theta_{\lambda}^{4}(0, s)-\lambda \theta_{q}^{4}(0, s)-(1-\lambda) \theta_{\bar{q}}^{4}(0, s)\right) \mathrm{d} s \geq 0
\end{aligned}
$$

(recall that $\sigma \varepsilon(t)>0$ ) in contradiction with (4.5). We conclude that, for all $t \in(0, T)$, there exists at least one $s \in(0, t)$ such that $W(0, s)>0$. Taking $t>0$ arbitrarily small, we conclude that the function $t \rightarrow W(y=0, t)$ increases in a neighborhood of zero. Then, since $W(y=0, t) \in C([0, T])$, there exists a time $t_{1}>0$ such that $W(0, s)>0$ for all $s \in\left(0, t_{1}\right)$. If $t_{1}=T$, the proof is finished. If $t_{1}<T$, there exists a time $t_{1}^{+}>t_{1}$ such that $W\left(t_{1}^{+}\right)=0$. Then repeating the previous argument on the interval $\left[t_{1}^{+}, t\right]$ for any $t \in\left(t_{1}^{+}, T\right)$, we conclude that in the neighborhood of $t_{1}^{+}$, the function $W(0, \cdot)$ is strictly positive. Consequently, the points in $[0, T]$ where $W(0, \cdot)$ vanishes correspond to global minimum: for all $t \in[0, T], W(0, t) \geq 0$ and the convexity of $K$ follows.

Theorem 4.3. The extremal problem $\left(\mathcal{P}_{\psi}\right)$ admits a unique solution in $\mathcal{D}$.

Proof. This results from the fact that function $J_{\psi}$ is lower semi-continuous, strictly convex, over the closed convex set $\mathcal{D}$.

It results that the equivalent optimal control problem (4.1) is well-posed as well. From a practical viewpoint, it is convenient to address this problem with a penalty method. For any parameter $\epsilon>0$, we introduce the penalized extremal problem:

$$
\left(\mathcal{P}_{\epsilon}\right): \quad \inf _{q \in \mathcal{D}} J_{\alpha, \epsilon}(q):=\frac{1}{2}\|q\|_{L^{1}(0, T)}^{2}+\frac{\alpha}{2}\|q\|_{H^{1}(0, T)}^{2}+\frac{\epsilon^{-1}}{2}\left\|(\theta(0, \cdot)-\underline{\theta})^{-}\right\|_{L^{2}(0, T)}^{2}
$$

where $\mathcal{D}$ is defined in (4.2).

Theorem 4.4. For all $\epsilon>0$, Problem $\left(\mathcal{P}_{\epsilon}\right)$ admits a unique solution $q_{\epsilon}$. Moreover, as $\epsilon \rightarrow 0$, $q_{\epsilon}$ strongly converges in $H^{1}(0, T)$ to $q$, the solution of (4.1).

Proof. The functional $J_{\alpha, \epsilon}$ is strictly convex, satisfies the property $\lim _{\|q\|_{H^{1}(0, T)} \rightarrow+\infty} J_{\alpha, \epsilon}(q)=+\infty$ and is continuous over $H^{1}(0, T)$ in view of remark (3.3). Moreover, the set $\mathcal{D}$ is a closed convex set of $H^{1}(0, T)$ which gives the existence and uniqueness of a solution $q_{\epsilon}$ of Problem $\left(\mathcal{P}_{\epsilon}\right)$. The strong convergence of $q_{\epsilon}$ with respect to $\epsilon$ is the consequence of the strict convexity of $J_{\alpha, \epsilon}$. In view of Theorem (3.2), this strong convergence implies the convergence of the corresponding solution $\theta_{\epsilon}=\theta\left(q_{\epsilon}\right)$ toward the solution $\theta(q)$ in $C([0, T], V)$. 


\subsection{Optimality system for the penalized extremal problem}

We derive in this section the optimality condition associated to the extremal problem $\left(\mathcal{P}_{\epsilon}\right)$. This allows to determine the first order variation of the cost $J_{\alpha, \epsilon}$ and define a minimizing sequence.

Let $H_{0,0}^{1}(0, T)=\left\{q \in H^{1}(0, T), q(0)=0\right\}$.

Theorem 4.5. For any $\alpha>0, \epsilon>0$, the functional $J_{\alpha, \epsilon}$ is Gâteaux differentiable on the set $\mathcal{D}$ and its derivative at $q \in \mathcal{D}$ in the admissible direction $\bar{q}$ (i.e. $\bar{q} \in H_{0,0}^{1}(0, T)$ such that $q+\eta \bar{q} \in \mathcal{D}$ for all $\eta \neq 0$ small) is given by

$$
\begin{aligned}
<J_{\alpha, \epsilon}^{\prime}(q), \bar{q}> & :=\lim _{\eta \rightarrow 0} \frac{J_{\alpha, \epsilon}(q+\eta \bar{q})-J_{\alpha, \epsilon}(q)}{\eta} \\
& =\int_{0}^{T}\left(\|q\|_{L^{1}(0, T)}-p\left(y_{0}, \cdot\right)\right) \bar{q} \mathrm{~d} t+\alpha \int_{0}^{T}\left(q \bar{q}+q_{t} \bar{q}_{t}\right) \mathrm{d} t
\end{aligned}
$$

where $p$ solves the adjoint problem

$$
\begin{cases}-c(y) p_{t}(y, t)-\left(k(y) p_{y}(y, t)\right)_{y}=0, & (y, t) \in Q_{T}, \\ -k(0) p_{y}(0, t)=-f_{2}(t) p(0, t)-4 \sigma \varepsilon(t) \theta_{q}(0, t)^{3} p(0, t)-\epsilon^{-1}\left(\theta_{q}(0, t)-\underline{\theta}\right)^{-}, & t \in(0, T), \\ p_{y}\left(h_{e}, t\right)=0, & t \in(0, T), \\ p(y, T)=0, & y \in\left(0, h_{e}\right),\end{cases}
$$

and $\theta_{q}$ solves (2.4). As a consequence, the unique minimizer $q_{\epsilon}$ in the convex set $\mathcal{D}$ of the convex functional $J_{\alpha, \epsilon}$ is characterized by the optimality condition

$$
<J_{\alpha, \epsilon}^{\prime}\left(q_{\epsilon}\right), \bar{q}-q_{\epsilon}>\quad \geq 0, \quad \forall \bar{q} \in \mathcal{D} .
$$

Proof. Let $\theta_{q+\eta \bar{q}}$ be the solution associated to the control function $q+\eta \bar{q}$. Using that the function $x \rightarrow(x-\underline{\theta})^{-}$ is 1 -Lipschitz on $\mathbb{R}$, we write that

$$
\begin{aligned}
\int_{0}^{T}\left(\left(\theta_{q+\eta \bar{q}}(0, t)-\underline{\theta}\right)^{-}\right)^{2} \mathrm{~d} t & -\int_{0}^{T}\left(\left(\theta_{q}(0, t)-\underline{\theta}\right)^{-}\right)^{2} \mathrm{~d} t=\int_{0}^{T}\left(\left(\theta_{q+\eta \bar{q}}(0, t)-\underline{\theta}\right)^{-}-\left(\theta_{q}(0, t)-\underline{\theta}\right)^{-}\right) \\
\times & \left(\left(\theta_{q+\eta \bar{q}}(0, t)-\underline{\theta}\right)^{-}+\left(\theta_{q_{s}}(0, t)-\underline{\theta}\right)^{-}\right) \mathrm{d} t \\
\leq & \int_{0}^{T}\left|\theta_{q+\eta \bar{q}}(0, t)-\theta_{q}(0, t)\right|\left(\left(\theta_{q+\eta \bar{q}}(0, t)-\underline{\theta}\right)^{-}+\left(\theta_{q}(0, t)-\underline{\theta}\right)^{-}\right) \mathrm{d} t \\
\leq & \left\|\theta_{q+\eta \bar{q}}(0, \cdot)-\theta_{q}(0, \cdot)\right\|_{L^{2}(0, T)}\left(\left\|\left(\theta_{q+\eta \bar{q}}(0, \cdot)-\underline{\theta}\right)^{-}\right\|_{L^{2}(0, T)}\right. \\
& \left.+\left\|\left(\theta_{q}(0, \cdot)-\underline{\theta}\right)^{-}\right\|_{L^{2}(0, T)}\right) .
\end{aligned}
$$

Estimate (3.18) implies that $\left\|\theta_{q+\eta \bar{q}}(0, \cdot)-\theta_{q}(0, \cdot)\right\|_{L^{2}(0, T)} \leq\left\|\theta_{q+\eta \bar{q}}-\theta_{q}\right\|_{L^{2}(0, T, V)} \leq \eta\|\bar{q}\|_{L^{2}(0, T)}$ and that

$$
\lim _{\eta \rightarrow 0} \frac{\int_{0}^{T}\left(\left(\theta_{q+\eta \bar{q}}(0, t)-\underline{\theta}\right)^{-}\right)^{2} \mathrm{~d} t-\int_{0}^{T}\left(\left(\theta_{q}(0, t)-\underline{\theta}\right)^{-}\right)^{2} \mathrm{~d} t}{\eta} \leq 2\|\bar{q}\|_{L^{2}(0, T)}\left\|\left(\theta_{q}(0, \cdot)-\underline{\theta}\right)^{-}\right\|_{L^{2}(0, T)} .
$$

Let us now expand the solution $\theta_{q+\eta \bar{q}}$ as follows $\theta_{q+\eta \bar{q}}=\theta_{q}+\eta \theta_{\eta}$ where $\theta_{q}$ solves (2.4) and $\theta_{\eta}$ solves

$$
\begin{cases}c(y) \theta_{\eta, t}(y, t)-\left(k(y) \theta_{\eta, y}(y, t)\right)_{y}=\bar{q}(t) \delta_{y_{0}}(y), & (y, t) \in Q_{T}, \\ -k(0) \theta_{\eta, y}(0, t)=-f_{2}(t) \theta_{\eta}(0, t)-4 \sigma \varepsilon(t) \theta_{q}(0, t)^{3} \theta_{\eta}(0, t)-\eta \sigma \varepsilon(t) f\left(t, \eta, \theta_{q}, \theta_{\eta}\right), & t \in(0, T), \\ \theta_{\eta, y}\left(h_{e}, t\right)=0, & t \in(0, T), \\ \theta_{\eta}(y, 0)=0, & y \in\left(0, h_{e}\right)\end{cases}
$$


with $f\left(t, \eta, \theta_{q}, \theta_{\eta}\right):=6 \theta_{q}^{2}(0, t) \theta_{\eta}^{2}(0, t)+4 \eta \theta_{q}(0, t) \theta_{\eta}^{3}(0, t)+\eta^{2} \theta_{\eta}^{4}(0, t)$. In particular, (3.18) implies the uniform estimate $\left\|\theta_{\eta}\right\|_{L^{2}(0, T, V)} \leq C\|\bar{q}\|_{L^{2}(0, T)}$. The compact injection of $V$ in $C\left(\left[0, h_{e}\right]\right)$ then allows to show that the term $\left\|\theta_{\eta}(0, \cdot)-\hat{\theta}_{\bar{q}}(0, \cdot)\right\|_{L^{2}(0, T)}$ converges to 0 as $\eta \rightarrow 0$ where $\hat{\theta}_{\bar{q}}$ solves the linear boundary value problem

$$
\begin{cases}c(y) \hat{\theta}_{\bar{q}, t}(y, t)-\left(k(y) \theta_{\bar{q}, y}(y, t)\right)_{y}=\bar{q}(t) \delta_{y_{0}}(y), & (y, t) \in Q_{T}, \\ -k(0) \hat{\theta}_{\bar{q}, y}(0, t)=-f_{2}(t) \hat{\theta}_{\bar{q}}(0, t)-4 \sigma \varepsilon(t) \theta_{q}(0, t)^{3} \hat{\theta}_{\bar{q}}(0, t), & t \in(0, T), \\ \hat{\theta}_{\bar{q}, y}\left(h_{e}, t\right)=0, & t \in(0, T), \\ \hat{\theta}_{\bar{q}}(y, 0)=0, & y \in\left(0, h_{e}\right) .\end{cases}
$$

Altogether, we obtain that

$$
\begin{aligned}
\lim _{\eta \rightarrow 0} \frac{J_{\alpha, \epsilon}(q+\eta \bar{q})-J_{\alpha, \epsilon}(q)}{\eta}= & \|q\|_{L^{1}(0, T)} \int_{0}^{T} \bar{q} \mathrm{~d} t \\
& +\alpha \int_{0}^{T}\left(q \bar{q}+q_{t} \bar{q}_{t}\right) \mathrm{d} t+\epsilon^{-1} \int_{0}^{T}\left(\theta_{q}(0, \cdot)-\underline{\theta}\right)^{-} \hat{\theta}_{\bar{q}}(0, t) \mathrm{d} t .
\end{aligned}
$$

Eventually, using that the adjoint solution $p$ defined by (4.7) solves equivalently the variational formulation

$$
\begin{aligned}
-\left(c p_{t}(t), \phi\right)_{H}+a(t, p(t), \phi) & +4 \sigma \varepsilon(t) \theta_{q}(0, t)^{3} p(0, t) \phi(0) \\
& =-\epsilon^{-1}\left(\theta_{q}(0, t)-\underline{\theta}\right)^{-} \phi(0) \quad \forall \phi \in V, \text { a.e.t } \in(0, T),
\end{aligned}
$$

and

$$
p(T)=0,
$$

we obtain, taking $\phi=\hat{\theta}_{\bar{q}}(\cdot, t)$ in $(4.10)$ and $\phi=p(\cdot, t)$ in $(3.2)$ that

$$
\epsilon^{-1} \int_{0}^{T}\left(\theta_{q}(0, \cdot)-\underline{\theta}\right)^{-} \hat{\theta}_{\bar{q}}(0, \cdot) \mathrm{d} t=-\int_{0}^{T} p\left(y_{0}, \cdot\right) \bar{q} \mathrm{~d} t
$$

leading to (4.6).

Remark 4.6. Writing that $\sigma \varepsilon(\cdot) \theta_{q}(0, \cdot)^{3} \in L^{\infty}(0, T)$ and that $-\epsilon^{-1}\left(\theta_{q}(0, \cdot)-\underline{\theta}\right)^{-} \in L^{2}(0, T)$, we obtain, following the steps 1 and 2 of the proof of Theorem 3.2, that the adjoint variable $p$ solution of the linear weak formulation (4.10) and (4.11) is unique and satisfies the regularity $p \in L^{\infty}(0, T ; H) \cap L^{2}(0, T ; V)$. Consequently, $p\left(y_{0}, \cdot\right)$ is in $L^{2}(0, T)$ and the first integral in (4.6) is well-defined.

We now define the function $\hat{q} \in H_{0,0}^{1}(0, T)$ as the unique solution of the formulation:

$$
\int_{0}^{T}\left(\|\hat{q}\|_{L^{1}(0, T)} \bar{q}+\alpha\left(\hat{q} \bar{q}+\hat{q}_{t} \bar{q}_{t}\right)\right) \mathrm{d} t=<J_{\alpha, \epsilon}^{\prime}(q), \bar{q}>, \quad \forall \bar{q} \in H_{0,0}^{1}(0, T)
$$

so that $\left\langle J_{\alpha, \epsilon}^{\prime}(q), \hat{q}>=\|\hat{q}\|_{L^{1}(0, T)}^{2}+\alpha\|\hat{q}\|_{H_{0}^{1}(0, T)}^{2}\right.$ is nonnegative. This property allows to set up a fixed step gradient projection algorithm: given $q^{0} \in \mathcal{D}, \eta>0$ small enough, compute iteratively with respect to $k$ the sequence $q^{k} \in \mathcal{D}$ as follows

$$
q^{k+1}=P_{\mathbb{R}^{+}}\left(q^{k}-\eta J^{\prime}\left(q^{k}\right)\right), \quad k \geq 0
$$

so that $\left(q^{k}\right)_{k \in \mathbb{N}}$ is a minimizing sequence for $J_{\alpha, \epsilon}$. Equation (4.12) can be decomposed into the following steps: given $q^{k} \in \mathcal{D}$,

- Compute $\hat{q}^{k} \in H_{0,0}^{1}(0, T)$ unique solution of

$$
\int_{0}^{T}\left(\left\|\hat{q}^{k}\right\|_{L^{1}(0, T)} \bar{q}+\alpha\left(\hat{q}^{k} \bar{q}+\left(\hat{q}^{k}\right)_{t} \bar{q}_{t}\right)\right) \mathrm{d} t=<J_{\alpha, \epsilon}^{\prime}\left(q^{k}\right), \bar{q}>, \quad \forall \bar{q} \in H_{0,0}^{1}(0, T) ;
$$


- Set $q^{k+1 / 2}:=q^{k}-\eta \hat{q}^{k} \in H_{0,0}^{1}(0, T)$ and then $q^{k+1}:=\max \left(0, q^{k+1 / 2}\right) \in \mathcal{D}$.

Remark 4.7. Equation (4.13) is equivalent to non local differential equation

$$
\left\{\begin{array}{l}
\alpha\left(\hat{q}-\hat{q}_{t t}\right)+\|\hat{q}\|_{L^{1}(0, T)}=\alpha\left(q-q_{t t}\right)+\|q\|_{L^{1}(0, T)}-p\left(y_{0}, \cdot\right), \quad t \in(0, T), \\
\hat{q}(0)=0, \hat{q}_{t}(T)=q_{t}(T) .
\end{array}\right.
$$

Remark that actually we may drop the $L^{1}$-norm in the left hand side: since $H^{1}(0, T) \subset L^{1}(0, T)$, the resulting sequence is still in $\mathcal{D}$. Moreover, we may impose the Dirichlet assumption $\hat{q}^{n}(T)=0 ;(4.13)$ is unchanged except that the space for the test functions $\bar{q}$ is then $H_{0}^{1}(0, T)$.

\section{Approximation of the optimal CONTRol Problem AND EXPERIMents}

\subsection{Numerical approximation}

The resolution of the variational formulations (3.2) and (4.10) is performed using a finite element approximation with respect to the space variable $y$ and a finite difference approximation with respect to the time variable $t$. For convenience, we replace the Neumann boundary condition at $y=h_{e}$ by a Dirichlet condition (see Rem. 3.4). Let $N_{y}$ a positive integer and $\left(x_{i}\right)_{i=1, \ldots, N_{y}}$ a subdivision of the interval $\left[0, h_{e}\right]$ such that $y_{0}=0$, $y_{N_{y}}=h_{e}$ and $y_{i}<y_{i+1}$ for all $i$ and $\left[0, h_{e}\right]=\cup_{i=0}^{N_{y}-1}\left[y_{i}, y_{i+1}\right]$. We note $h=\max _{i}\left|y_{i+1}-y_{i}\right|$. We then introduce the following conformal finite element approximation $V_{h}$ of $V$ :

$$
V_{h}=\left\{\theta_{h} \in C^{1}\left(\left[0, h_{e}\right]\right), \theta_{\left.h\right|_{\left[x_{i}, x_{i+1}\right]}} \in \mathbb{P}_{1} \quad \forall i=1, \ldots, N_{y}-1\right\}
$$

where $\mathbb{P}_{1}$ denotes the space of polynomial functions of order one. We also consider, for some $\theta_{d} \in \mathbb{R}$ the space $V_{h}^{d}=\left\{\theta_{h} \in V_{h},\left(\theta_{h}\right)\left(h_{e}\right)=\theta_{d}\right\}$. The weak formulation associated to (2.4) (see (3.2) and (3.3)) is then approximated as follows: find $\theta_{h} \in L^{2}\left(0, T, V_{h}^{d}\right)$ such that

$$
\begin{aligned}
\left(c \theta_{h, t}(t), \phi_{h}\right)_{H}+a\left(t, \theta_{h}(t), \phi_{h}\right) & +\sigma \varepsilon(t) \theta_{h}(0, t)^{4} \phi_{h}(0) \\
& =q(t) \phi_{h}\left(y_{0}\right)+f_{1}(t) \phi_{h}(0), \quad \forall \phi_{h} \in V_{h}, \text { a.e.t } \in(0, T)
\end{aligned}
$$

and

$$
\theta_{h}(0)=\pi_{h}\left(\theta_{0}\right)
$$

where $\pi_{h}: V \rightarrow V_{h}$ is the projection operator over $V_{h}$. Similarly, let $N_{t}$ be a positive integer and $\left(t_{n}\right)_{n=1, \ldots, N_{t}}$ a uniform subdivision of the time interval $[0, T]$ such that $t_{0}=0, t_{N_{t}}=T$ and $t_{n}=n \Delta t$ for all $n$ and $[0, T]=\cup_{n=0}^{N_{t}-1}\left[t_{n}, t_{n+1}\right]$. We note by $\left(\theta_{h}^{n}\right)$ an approximation of $\theta_{h}\left(\cdot, t_{n}\right)$ the solution of the following implicit Euler type scheme:

$$
\left\{\begin{array}{l}
\theta_{h}^{0}=\pi_{h}\left(\theta_{0}\right), \\
\left(c \frac{\theta_{h}^{n+1}-\theta_{h}^{n}}{\Delta t}, \phi_{h}\right)_{H}+a\left(t_{n+1}, \theta_{h}^{n+1}, \phi_{h}\right)+4 \sigma \varepsilon\left(t_{n+1}\right)\left(\theta_{h}^{n}(0)\right)^{3} \theta_{h}^{n+1}(0) \phi_{h}(0) \\
\quad-3 \sigma \varepsilon\left(t_{n}\right)\left(\theta_{h}^{n}(0)\right)^{4} \phi_{h}(0)=q\left(t_{n+1}\right) \phi_{h}\left(y_{0}\right)+f_{1}\left(t_{n+1}\right) \phi_{h}(0), \quad \forall \phi_{h} \in V_{h}, n \geq 0 .
\end{array}\right.
$$

In particular, the nonlinear term $\theta_{h}^{4}\left(t_{n+1}\right)$ is approximated as follows:

$$
\theta_{h}^{4}\left(t_{n+1}\right)=4 \theta_{h}^{3}\left(t_{n}\right) \theta_{h}\left(t_{n+1}\right)-3 \theta_{h}^{4}\left(t_{n}\right)+\mathcal{O}(\Delta t), \quad \forall n .
$$

In the sequel, we define $\Theta_{h}=\left\{\theta_{h}^{n}\right\}_{n=0, \ldots, N_{t}} \in\left(V_{h}\right)^{N_{t}+1}$. The extremal problem $\left(\mathcal{P}_{\epsilon}\right)$ is then approximated by the following one:

$$
\left(\mathcal{P}_{\epsilon, h, \Delta t}\right): \quad \inf _{q_{\Delta t} \in \mathcal{D}_{\Delta t}} J_{\alpha, \epsilon}\left(q_{\Delta t}\right):=\frac{1}{2}\left\|q_{\Delta t}\right\|_{L^{1}(0, T)}^{2}+\frac{\alpha}{2}\left\|q_{\Delta t}\right\|_{H^{1}(0, T)}^{2}+\frac{\epsilon^{-1}}{2}\left\|\left(\pi_{\Delta t}\left(\Theta_{h}(0)\right)(t)-\underline{\theta}\right)^{-}\right\|_{L^{2}(0, T)}^{2} .
$$


Here, $\Pi_{\Delta t}\left(\Theta_{h}(0)\right)(t)$ is the piecewise affine function such that $\Pi_{\Delta t}\left(\Theta_{h}(0)\right)\left(t^{n}\right)=\theta_{h}^{n}(0)$ for all $n$ where $\left(\theta_{h}^{n}\right)$ solves (5.3) and

$$
\mathcal{D}_{\Delta t}:=\left\{q_{\Delta t} \in H^{1}(0, T), q_{s, \Delta t}(0)=0,\left.q_{\Delta t}\right|_{\left[t_{n}, t_{n+1}\right]} \in \mathbb{P}_{1}, q_{\Delta t}\left(t_{n}\right) \geq 0, \forall n \geq 0\right\} .
$$

The extremal point of the functional $J_{\alpha, \epsilon}$ in $\mathcal{D}_{\Delta t}$ is determined by using the gradient projection algorithm described in the previous section. In particular, the adjoint problems (4.10) and (4.11) is approximated as follows:

$$
\left\{\begin{array}{l}
p_{h}^{N_{t}}=0, \\
-\left(c \frac{p_{h}^{n+1}-p_{h}^{n}}{\Delta t}, \phi_{h}\right)_{H}+a\left(t_{n}, p_{h}^{n}, \phi_{h}\right)+4 \sigma \varepsilon\left(t_{n}\right)\left(\theta_{h}^{n}(0)\right)^{3} p_{h}^{n}(0) \phi_{h}(0) \\
=-\epsilon^{-1}\left(\theta_{h}^{n}(0)-\underline{\theta}\right)^{-} \phi_{h}(0), \quad \forall \phi_{h} \in V_{h}, n \geq 0 .
\end{array}\right.
$$

\subsection{Presentation of the experimental data and initial condition}

We use real data obtained from measurements on the French highway A75 in Cantal (1100 m altitude) from october 2009 to march 2010. Measurements are made each hour and allow to compute the time functions $f_{1}$ and $f_{2}$ defined in (2.6). Figure 4 depicts these functions.

For the other parameters, we use numerical values obtained from experimental validations described in [2]: precisely, the albedo of the road surface is $A=0.08$ (used in $f_{1}$ ), while for the emissivity function of the road, we use the constant value $\varepsilon(t)=0.92$. Although the measurements of $\varepsilon$ show a time variability and a sensitivity of the road surface temperature $\theta(0, \cdot)$ w.r.t. $\varepsilon$ (see Fig. 5), the lack of emissivity measurements over a long period requires to identify and use a constant value $\varepsilon=0.92$. As discussed in [2], this identification leads to a good agreement between simulated and measured temperatures. From a theoretical viewpoint, remark that Theorem 3.2 is valid for a time-dependent emissivity function.

As described in Figure 3, the road is composed of 4 layers of depth $e_{1}=0.06 \mathrm{~m}, e_{2}=0.08 \mathrm{~m}, e_{3}=0.05 \mathrm{~m}$ and $e_{4}=h_{e}-e_{1}+e_{2}+e_{3}=14.81 \mathrm{~m}$, respectively. The total height of the road structure is $h_{e}=15 \mathrm{~m}$. The specific heat function $c$ and the thermal conductivity function $k$ are constant on each layer and takes the following values [2]:

$$
(c(y), k(y))= \begin{cases}\left(c_{1}, k_{1}\right)=(2144309,2.34), & y \in\left(0, e_{1}\right), \\ \left(c_{2}, k_{2}\right)=(1769723,1.56), & y \in\left(e_{1}, e_{1}+e_{2}\right), \\ \left(c_{3}, k_{3}\right)=(2676728,1.76), & y \in\left(e_{1}+e_{2}, e_{1}+e_{2}+e_{3}\right), \\ \left(c_{4}, k_{4}\right)=(1947505,2.08), & y \in\left(e_{1}+e_{2}+e_{3}, h_{e}\right) .\end{cases}
$$

Moreover, the initial condition $\theta_{0}$ is a priori not determined by experiments. We define the initial condition as the solution of the stationary model and satisfying the compatibility conditions (3.6) of Theorem 3.2: more precisely, we consider the solution of the boundary value problem:

$$
\begin{cases}-\left(k(y) \theta_{0, y}(y)\right)_{y}=0, & y \in\left(0, h_{e}\right) \\ -k(0) \theta_{0, y}(0)=f_{1}(0)-f_{2}(0) \theta_{0}(0)-\sigma \varepsilon(0) \theta_{0}(0)^{4}, & \theta_{0}\left(h_{e}\right)=\theta_{d} .\end{cases}
$$

Here, we have replaced the homogeneous Neumann boundary condition $\theta_{0, y}\left(h_{e}\right)=0$ by a Dirichlet condition $\theta(L)=\theta_{d}$; the reason is that the temperature, at the height $h_{e}=15 \mathrm{~m}$ under the road surface, is time independent and close to a value equal to $15^{\circ} \mathrm{C}$ (equivalently $273.15+15^{\circ} \mathrm{K}$ ). The resolution of the nonlinear boundary value problem with $\theta_{d}=288.15$, using a Newton type algorithm and a finite element discretization as above, leads to a continuous function, affine on each layers, and increasing from the value $\theta_{0}(y=0) \approx K+6.29 \mathrm{~K}$ at the road surface to $\theta_{0}\left(y=h_{e}\right)=\theta_{d} \mathrm{~K}$. Moreover, by definition, this solution $\theta_{0}$ satisfies the assumption $\left(k\left(\theta_{0}\right)_{y}\right)_{y} \in V^{\prime}($ see $\mathcal{H})$, that is the jump $\left[k\left(\theta_{0}\right)_{y} \cdot \nu\right]$ is equal to zero at $y=e_{1}, e_{1}+e_{2}$ and at $y=e_{1}+e_{2}+e_{3}$. Eventually, we observe that the value $\theta_{0}(y=0) \approx 279.43 \mathrm{~K}$ as the initial temperature at the road surface is 

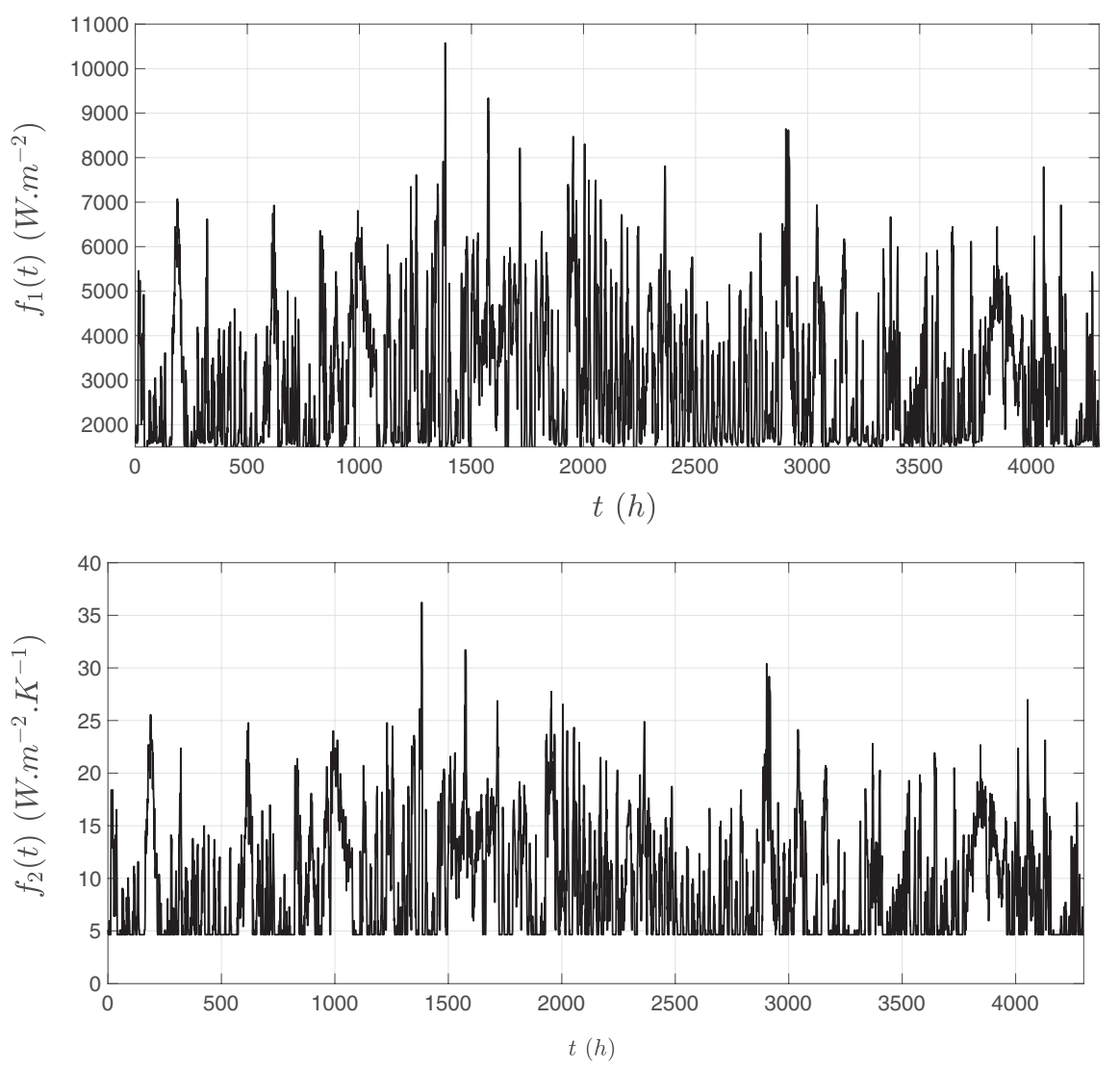

Figure 4. The time functions $f_{1}(t o p)$ and $f_{2}$ (bottom) defined in (2.6).

in agreement with typical measurements in october (we recall that our study is based on measurements from october 2009 to march 2010).

\subsection{Numerical experiments: optimal control minimizing $J_{\alpha, \epsilon}$}

We now discuss some numerical experiments associated to the optimal problem $\left(\mathcal{P}_{\epsilon}\right)$ : precisely, we minimize over $\mathcal{D}$ the functional

$$
J_{\alpha, \epsilon}(q):=\frac{1}{2}\|q\|_{L^{1}(0, T)}^{2}+\frac{\alpha}{2}\left(T\|q\|_{L^{2}(0, T)}^{2}+\frac{T^{3}}{4 \pi^{2}}\left\|q_{t}\right\|_{L^{2}(0, T)}^{2}\right)+\frac{\epsilon^{-1}}{2}\left\|\left(\theta_{q}(0, \cdot)-\underline{\theta}\right)^{-}\right\|_{L^{2}(0, T)}^{2} .
$$

It is necessary to adjust the constants in front of the norms in term of the value of the time interval $T$, which, expressed in seconds, is large: precisely $T=15494400 \mathrm{~s}$ (equivalently $4304 \mathrm{~h}$ ). We use for that the inequality $\|q\|_{L^{1}(0, T)} \leq \sqrt{T}\|q\|_{L^{2}(0, T)}$ and the Wirtinger inequality $\|q\|_{L^{2}(0, T)} \leq T /(2 \pi)\left\|q_{t}\right\|_{L^{2}(0, T)}$ (taking $q(0)=q(T)=0$ ). For the same reasons, coefficients must be properly chosen in the computation of the descent direction (see (4.13)): we use here

$$
\int_{0}^{T}\left(\hat{q}^{k} \bar{q}+T\left(\hat{q}^{k}\right)_{t} \bar{q}_{t}\right) \mathrm{d} t=<J_{\alpha, \epsilon}^{\prime}\left(q^{k}\right), \bar{q}>, \quad \forall \bar{q} \in H_{0,0}^{1}(0, T)
$$



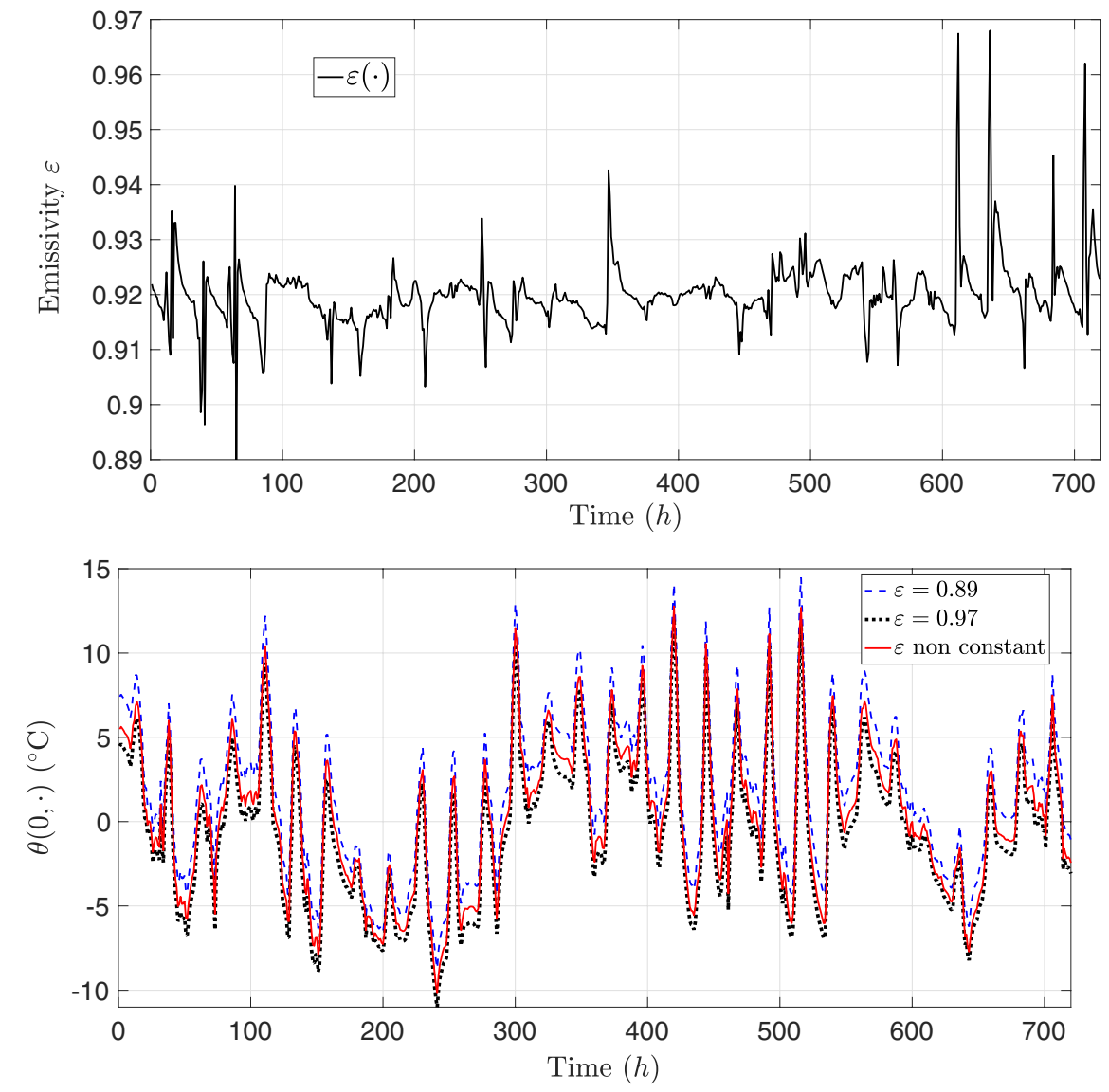

Figure 5. Measurements over one month of the emissivity $\varepsilon(t o p)$ and the road surface temperature $\theta(0, \cdot)$ for the time-dependent $\varepsilon$, and constant $\varepsilon$ 's respectively fixed to the minimal and maximal values (bottom).

Similarly, the penalty parameter $\epsilon$ is adjusted so that the optimal control leads to values of the same order for the norm $\left\|\left(\theta_{q}(0, \cdot)-\underline{\theta}\right)^{-}\right\|_{L^{2}(0, T)}$, independently of the value of the parameter $\alpha$, the value of the height $y_{0}$ (punctual support of the control $q$ ) and of the minimal temperature required $\underline{\theta}$.

The time discretization parameter $\Delta t$ is given by the data: $\Delta t=$ one hour over a period of $4304 \mathrm{~h}$. Scaled to a time interval of length one, $\Delta t=1 / 4304 \approx 2.32 \times 10^{-4}$. Concerning the approximation with respect to $y$, we use a nonuniform discretization of the interval $\left[0, h_{e}\right]$ : precisely, we use 50 finite elements in each layers of height $0.06,0.08,0.05$ and $14.81 \mathrm{~m}$, respectively. In the sequel, all iterative algorithms used to approximate the optimal controls are initialized with a zero source $q_{0}=0$ and stopped at iterate $k$ with the criterion $\left|J\left(q_{k}\right)-J\left(q_{k-1}\right)\right| / J\left(q_{0}\right) \leq 10^{-10}$, where $J$ is the minimized function and $q_{k}$ is the $k$ th iterate of the control.

In order to analyse the specific effect of the $L^{1}$-norm minimization in (5.6), we start by the standard minimization of the $L^{2}$-norm:

$$
\inf _{q \in L^{2}(0, T), q \geq 0} \widetilde{J}_{\epsilon}(q)
$$

where

$$
\widetilde{J}_{\epsilon}(q):=\frac{1}{2}\|q\|_{L^{2}(0, T)}^{2}+\frac{\epsilon^{-1}}{2}\left\|\left(\theta_{q}(0, \cdot)-\underline{\theta}\right)^{-}\right\|_{L^{2}(0, T)}^{2}
$$


TABLE 1. Numerical norms of the optimal control of problem (5.8) with respect to the parameter $\epsilon \in\left\{10^{-6}, 10^{-4}\right\}-y_{0}=0.1$.

\begin{tabular}{llllll}
\hline \hline$\epsilon$ & $10^{-6}$ & $4.10^{-6}$ & $10^{-5}$ & $5.10^{-5}$ & $10^{-4}$ \\
\hline$\|q\|_{L^{2}(0, T)}$ & $2.68 \times 10^{5}$ & $2.66 \times 10^{5}$ & $2.62 \times 10^{5}$ & $2.48 \times 10^{5}$ & $2.35 \times 10^{5}$ \\
$\|q\|_{L^{1}(0, T)}$ & $5.83 \times 10^{8}$ & $5.79 \times 10^{8}$ & $5.73 \times 10^{8}$ & $5.46 \times 10^{8}$ & $5.23 \times 10^{8}$ \\
$\|q\|_{L^{\infty}(0, T)}$ & $4.14 \times 10^{2}$ & $4.00 \times 10^{2}$ & $3.89 \times 10^{2}$ & $3.54 \times 10^{2}$ & $3.18 \times 10^{2}$ \\
$\left\|(\theta(0, \cdot)-\underline{\theta})^{-}\right\|_{L^{2}(0, T)}$ & $1.47 \times 10^{1}$ & $5.40 \times 10^{1}$ & $1.23 \times 10^{2}$ & $4.71 \times 10^{2}$ & $8.10 \times 10^{2}$ \\
$\left\|(\theta(0, \cdot)-\underline{\theta})^{-}\right\|_{L^{\infty}(0, T)}$ & $6.03 \times 10^{-2}$ & $1.90 \times 10^{-1}$ & $4.01 \times 10^{-1}$ & $1.11 \times 10^{0}$ & $1.72 \times 10^{0}$ \\
$\sharp$ iterates & 183 & 234 & 130 & 64 & 56 \\
\hline
\end{tabular}

TABLE 2. Numerical norms of the optimal control minimizing (5.6) with respect to the parameter $\alpha \in\left\{10^{-7}, 10^{-3}\right\}-y_{0}=0.1$.

\begin{tabular}{llllll}
\hline \hline$\alpha$ & $10^{-7}$ & $10^{-6}$ & $10^{-5}$ & $10^{-4}$ & $10^{-3}$ \\
\hline$\epsilon$ & $10^{-12}$ & $7.10^{-13}$ & $5.10^{-13}$ & $3.10^{-13}$ & $2.10^{-13}$ \\
\hline$\|q\|_{L^{2}(0, T)}$ & $2.97 \times 10^{5}$ & $2.86 \times 10^{5}$ & $2.81 \times 10^{5}$ & $2.93 \times 10^{5}$ & $3.17 \times 10^{5}$ \\
$\|q\|_{L^{1}(0, T)}$ & $4.46 \times 10^{8}$ & $4.65 \times 10^{8}$ & $5.26 \times 10^{8}$ & $6.20 \times 10^{8}$ & $7.67 \times 10^{8}$ \\
$|q|_{H^{1}(0, T)}$ & $2.85 \times 10^{1}$ & $1.61 \times 10^{1}$ & $7.40 \times 10^{0}$ & $3.10 \times 10^{0}$ & $1.49 \times 10^{0}$ \\
$\|q\|_{L^{\infty}(0, T)}$ & $5.06 \times 10^{2}$ & $4.06 \times 10^{2}$ & $3.58 \times 10^{2}$ & $3.18 \times 10^{2}$ & $2.85 \times 10^{2}$ \\
$\left\|(\theta(0, \cdot)-\underline{\theta})^{-}\right\|_{L^{2}(0, T)}$ & $4.09 \times 10^{1}$ & $3.88 \times 10^{1}$ & $4.32 \times 10^{1}$ & $4.52 \times 10^{1}$ & $6.07 \times 10^{1}$ \\
$\left\|(\theta(0, \cdot)-\underline{\theta})^{-}\right\|_{L^{\infty}(0, T)}$ & $9.33 \times 10^{-2}$ & $9.30 \times 10^{-2}$ & $1.35 \times 10^{-1}$ & $2.11 \times 10^{-1}$ & $4.32 \times 10^{-1}$ \\
$\sharp$ iterates & 2201 & 1345 & 1779 & 2441 & 12455 \\
\hline
\end{tabular}

We gather in Table 1 some numerical norms of the optimal control $q$ of (5.8) with respect to the penalty parameter $\epsilon$. The punctual control $q$ is located on the middle of the porous layer, i.e. at $y_{0}=0.1 \mathrm{~m}$.

The minimal temperature required along the six months period is $\underline{\theta}=275.15 \mathrm{~K}$ : we therefore consider a slightly larger value than 273.15 (corresponding to the $0^{\circ} \mathrm{C}$ ) in order to account for the possible errors in the experimental measurements and models. Remark that the initial condition $\theta_{0}$, solution of (5.5), satisfies this property. As expected, $\|q\|_{L^{2}(0, T)},\|q\|_{L^{1}(0, T)}$ and $\|q\|_{L^{\infty}(0, T)}$ increase and $\left\|(\theta(0, \cdot)-\underline{\theta})^{-}\right\|_{L^{\infty}(0, T)}$ decreases when $\epsilon$ decreases.

Table 2 collects some numerical norms of the optimal control $q$ (minimizer of the functional $J_{\alpha, \epsilon}$ ) with respect to the regularizing parameter $\alpha$. The penalty parameter $\epsilon$ is chosen so that, for each $\alpha$, the $L^{2}$-norm $\left\|(\theta(0, \cdot)-\underline{\theta})^{-}\right\|_{L^{2}(0, T)}$ takes approximatively the value $5 \times 10^{1}$, leading to a violation of the constraint $\theta(0, t) \geq$ $K+2$ of the order $10^{-1}$ degree (see $\left\|(\theta(0, \cdot)-\underline{\theta})^{-}\right\|_{L^{\infty}(0, T)}$ in Tab. 2).

As we can see in Figure 6, small values of $\alpha$ lead to sparse controls, with smaller $L^{1}$-norm and larger $L^{\infty}$-norm (see [6]). On the contrary, large values of $\alpha$ lead to more regular controls (see Tab. 2).

In order to avoid large punctual values for the optimal $L^{1}$-control, which may be not physical or unrealistic at the practical level, we may constrain its values in a given interval $[0, \lambda]$. Results in Section 4 may be easily adapted to this new situation. Figure 7 displays some optimal controls satisfying the additional condition $\|q\|_{L^{\infty}} \leq \lambda$ for $\lambda=200$ and 285 (the unbounded case $\lambda=+\infty$ is plotted as well for comparison).

We notice the effect of such boundedness on the sparsity of the control, which tends to decrease when $\lambda$ decreases, as we can see especially for $\lambda=200$. However, in this latter case, $\|q\|_{L^{1}(0, T)}=5.78 \times 10^{8}$, which is much higher than $4.63 \times 10^{8}$ or $4.46 \times 10^{8}$ corresponding respectively to $\lambda=285$ and $\lambda=+\infty$. Moreover, the value $\lambda=200$ is not large enough to satisfy the constraint at the surface of the road, since we obtain $\left\|(\theta(0, \cdot)-\underline{\theta})^{-}\right\|_{L^{\infty}(0, T)}$ of order 2.0, much larger to that corresponding to $\lambda=285$ and $\lambda=+\infty$ (lower than 


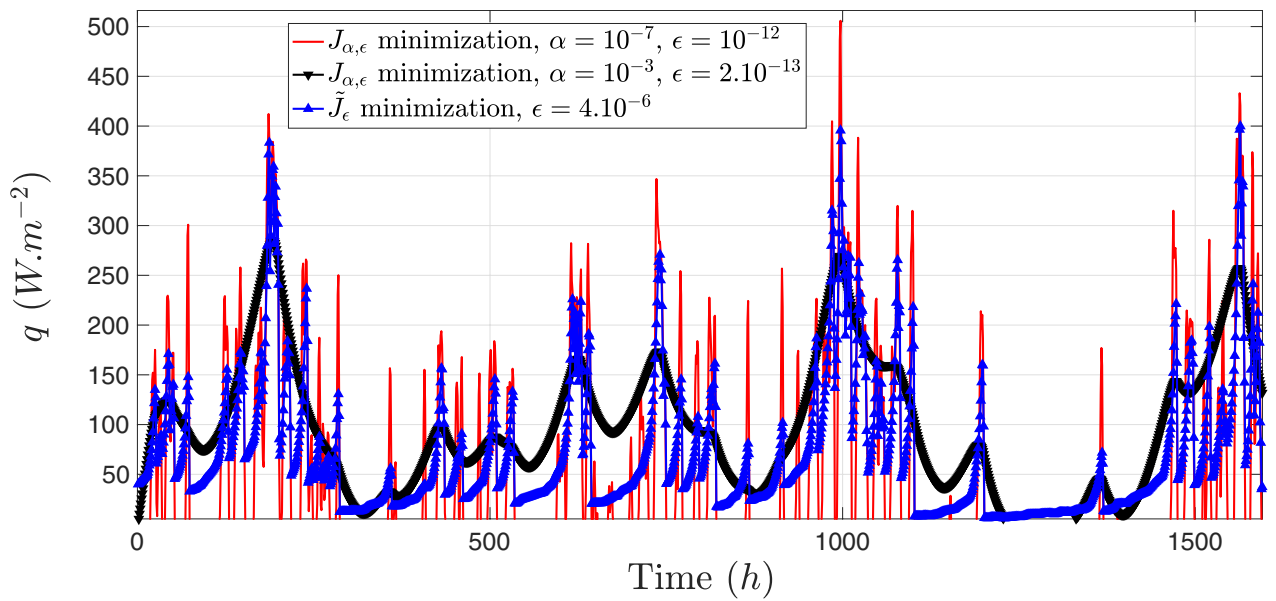

Figure 6. Optimal controls $q$ on $[0,1600]$ corresponding to the minimization of $J_{\alpha, \epsilon}$ and $\tilde{J}_{\epsilon}$.

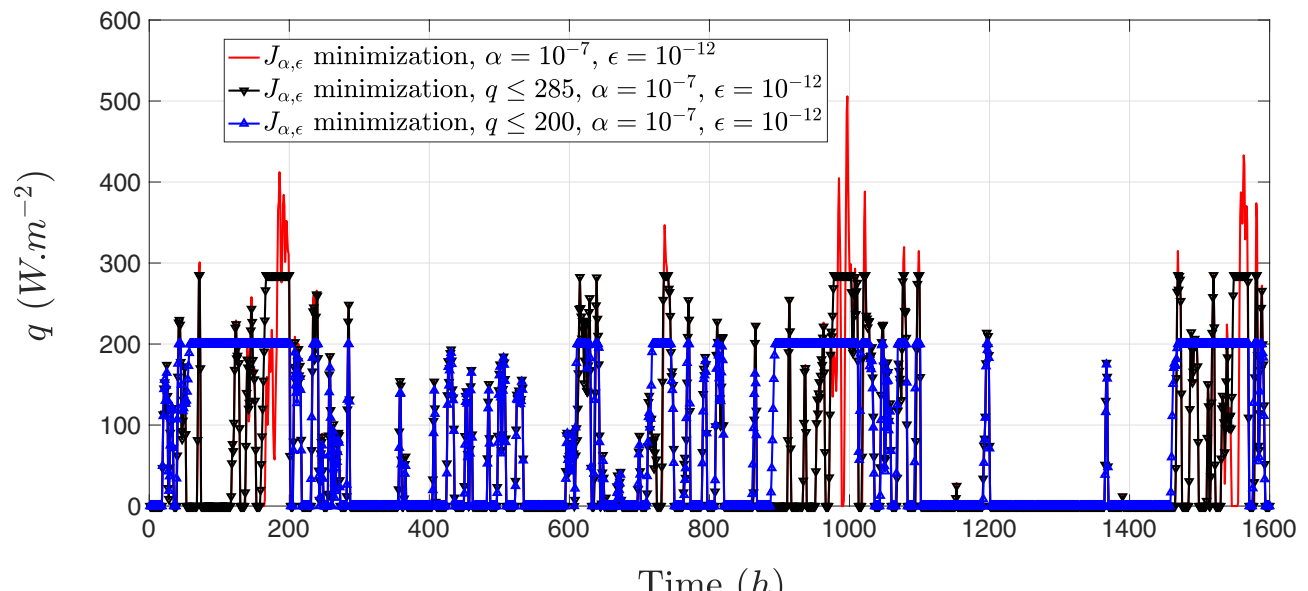

FiguRE 7. Optimal controls $q$ on $[0,1600]$ corresponding to the minimization of $J_{\alpha, \epsilon}$ under the additional constraint $\|q\|_{\infty} \leq \lambda$ for $\lambda=200$ and 285 .

0.2 , as we can see in Figure 8. A minimal value of $\|q\|_{L^{\infty}(0, T)}$ is thus necessary to maintain the road out of frost or snow (see Sect. 5.4 on bang-bang controls).

It is also interesting to study the local effect of control. As can be seen in Figure 9, the more the control is regular, the less it reacts instantaneously with respect to the uncontrolled surface temperature $\theta_{q=0}(0, \cdot)$.

\subsection{Bang-bang control}

We are now looking for so-called bang-bang controls: there are more convenient on a practical viewpoint as they take only a finite number of distinct values. Precisely, we assume that controls $q$ take the form

$$
q(t)=\lambda s(t), \quad \lambda \geq 0, s(t) \in\{0,1\}
$$

so that the control $q$ takes only two values, 0 or $\lambda$. Remark that such controls are only in $L^{\infty}(0, T)$ and therefore do not fall in the framework of Theorem 3.2. The sequel of this section is thus formal. For any $L \in(0,1)$, we 


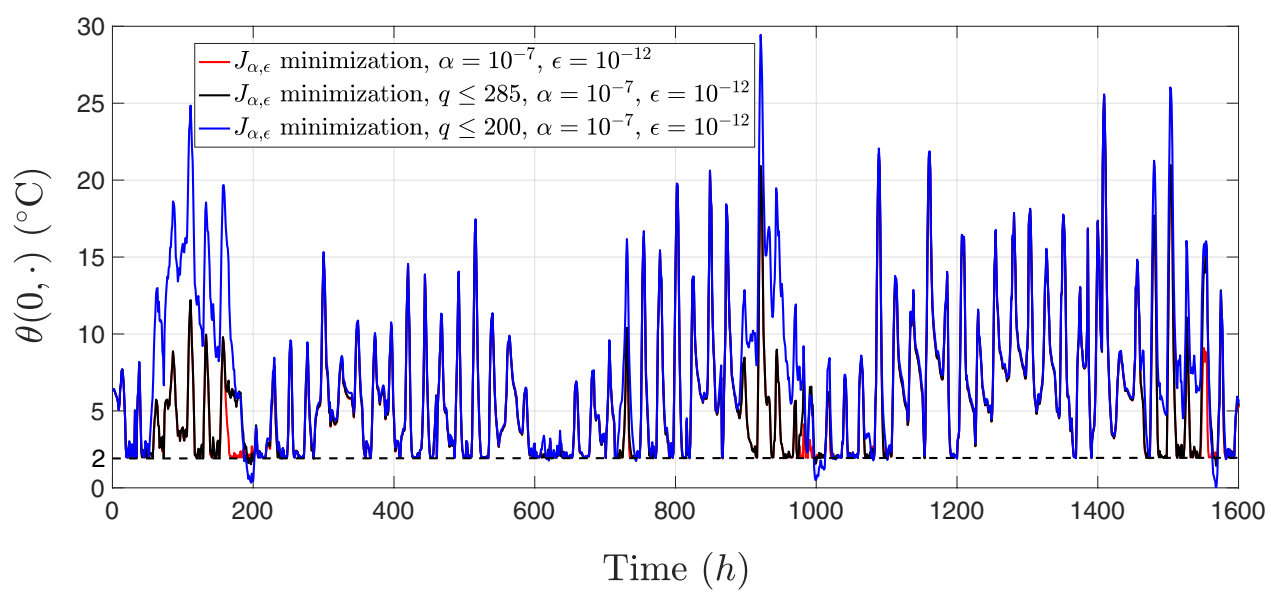

FIgURE 8 . Surface temperature $\theta(0, \cdot)$ on $[0,1600]$ corresponding to the minimization of $J_{\alpha, \epsilon}$ for different bounds of $\|q\|_{\infty}$.

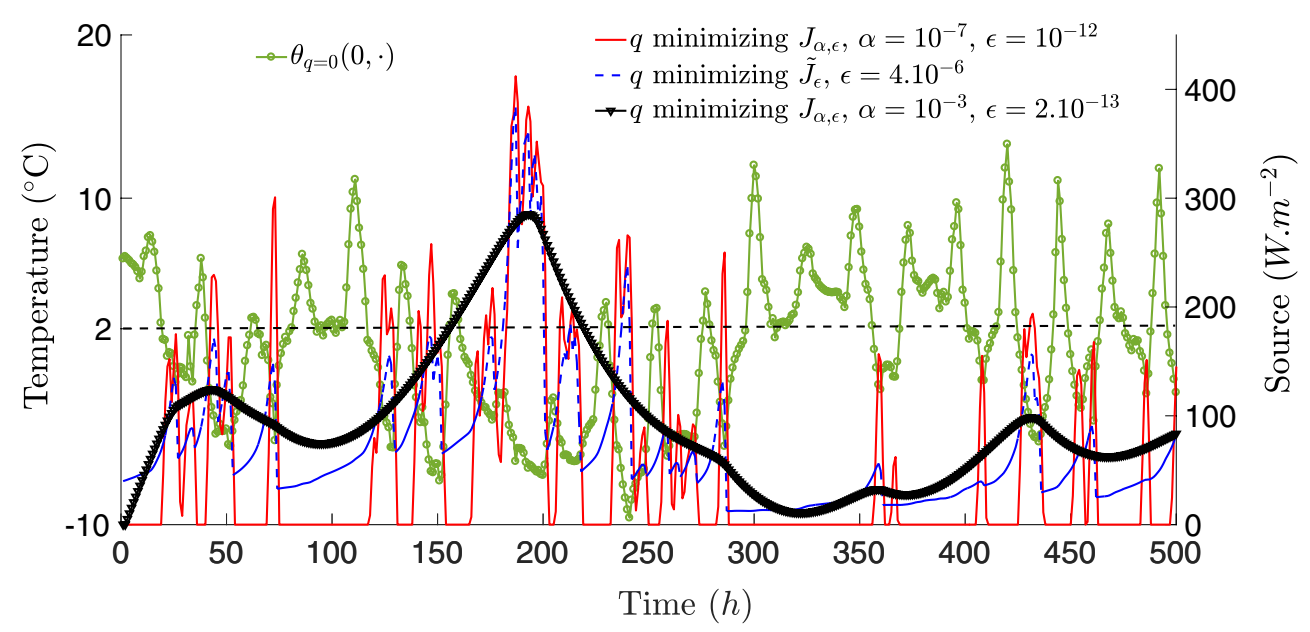

FigURE 9. Surface temperature $\theta_{q=0}(0, \cdot)$, and sources $q$ represented on $[0,500]$ corresponding to the minimization of $J_{\alpha, \epsilon}$ with $\alpha=10^{-3}$ and $\epsilon=2.10^{-13}, \alpha=10^{-7}$ and $\epsilon=10^{-12}$ and $\tilde{J}_{\epsilon}$ with $\epsilon=4 \cdot 10^{-6}$.

therefore consider formally the extremal problem

$$
\left\{\begin{array}{l}
\inf _{(\lambda, s) \in \mathcal{S}_{L}} J(\lambda, s)=\frac{1}{2} \lambda^{2} \\
\mathcal{S}_{L}:=\left\{\lambda \in \mathbb{R}^{+}, s \in L^{\infty}([0, T],\{0,1\}),\|s\|_{L^{1}(0, T)}=L T, \theta=\theta(q=\lambda s) \operatorname{solves}(2.4)\right\}
\end{array}\right.
$$

and minimize the amplitude $\lambda$ of the control $q$, assumed piecewise constant. A volume constraint through the parameter $L$ is introduced here to prevent the optimal control to be constant $q(t)=\lambda($ i.e. $s(t)=1)$ for all $t$. Adapting [16], we introduce two penalty parameters $\epsilon$ and $\epsilon_{1}$ for the constraints $s \in\{0,1\}$ and $\theta(0, t)-\underline{\theta}>0$, $t \in(0, T)$, respectively and a scalar Lagrange multiplier $\mu$ for the $L^{1}$-constraint on the density function $s$. This 
TABLE 3. Characteristics of the bang-bang controls with respect to $L$.

\begin{tabular}{llllll}
\hline \hline$L$ & $1 / 10$ & $1 / 5$ & $1 / 4$ & $1 / 3$ & 1 \\
\hline$\|q\|_{L^{1}(0, T)}=\lambda L T$ & $4.57 \times 10^{8}$ & $7.33 \times 10^{8}$ & $9.05 \times 10^{8}$ & $1.21 \times 10^{9}$ & $3.63 \times 10^{9}$ \\
$\|q\|_{L^{\infty}(0, T)}=\lambda$ & $2.95 \times 10^{2}$ & $2.37 \times 10^{2}$ & $2.34 \times 10^{-2}$ & $2.34 \times 10^{2}$ & $2.34 \times 10^{2}$ \\
$\left\|(\theta(0, \cdot)-\underline{\theta})^{-}\right\|_{L^{2}(0, T)}$ & $2.98 \times 10^{-1}$ & $2.21 \times 10^{-2}$ & $3.03 \times 10^{-3}$ & $1.74 \times 10^{-4}$ & $1.93 \times 10^{-3}$ \\
$\left\|(\theta(0, \cdot)-\underline{\theta})^{-}\right\|_{L^{\infty}(0, T)}$ & $3.65 \times 10^{-1}$ & $4.66 \times 10^{-2}$ & $2.16 \times 10^{-2}$ & $3.56 \times 10^{-6}$ & $4.01 \times 10^{-5}$ \\
$\sharp$ iterates & 456 & 361 & 312 & 197 & 102 \\
\hline
\end{tabular}

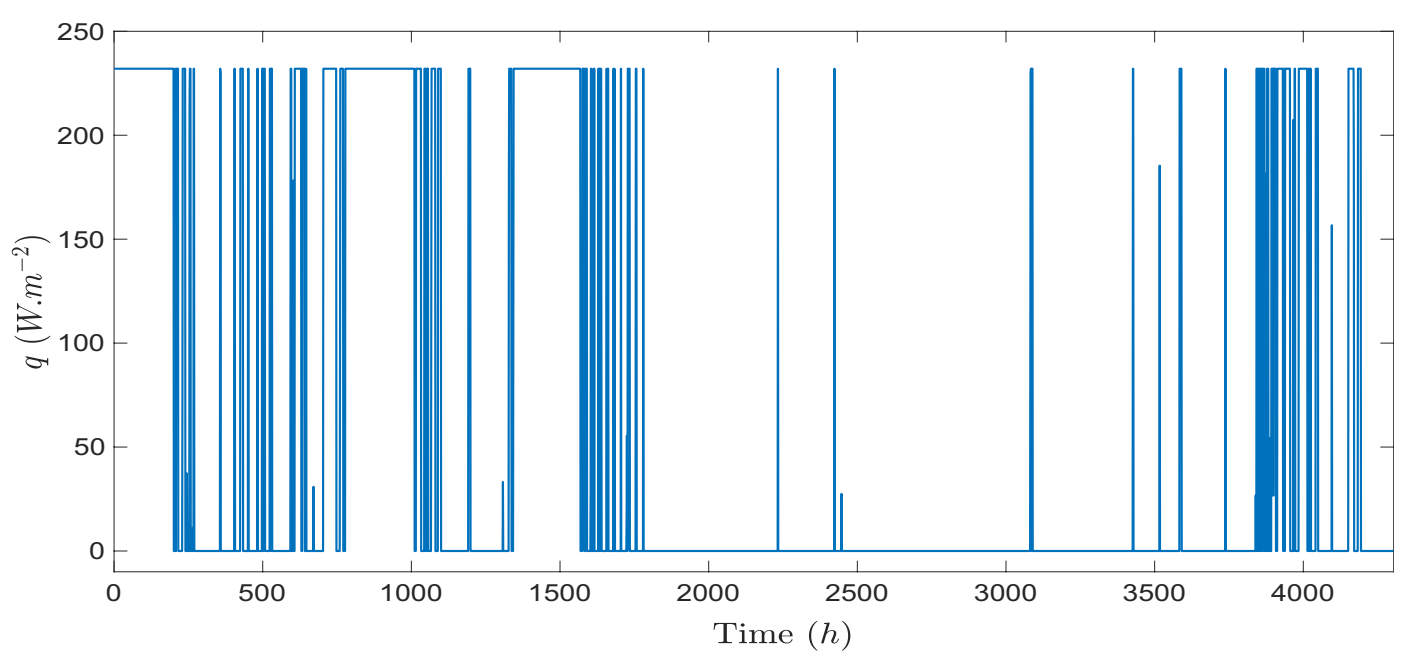

Figure 10. Optimal bang-bang control $q$ on $[0, T]$ corresponding to $L=1 / 4 . q(t)=\lambda s(t)$ with $\lambda \approx 2.34 \times 10^{2}$.

leads to the saddle-point problem

$$
\left\{\begin{array}{l}
\sup _{\mu \in \mathbb{R}} \inf _{(\lambda, s) \in \mathbb{R}^{+} \times L^{\infty}([0, T])} \mathcal{L}(s, \lambda, \mu):=J_{\epsilon, \epsilon_{1}}(s, \lambda)+\mu \int_{0}^{T}(s(t)-L) \mathrm{d} t, \\
J_{\epsilon, \epsilon_{1}}(s, \lambda):=\frac{\lambda^{2}}{2}+\frac{\epsilon^{-1}}{2} \int_{0}^{T}\left((\theta(0, t)-\underline{\theta})^{-}\right)^{2} \mathrm{~d} t+\epsilon_{1}^{-1} \int_{0}^{T} s(t)(1-s(t)) \mathrm{d} t
\end{array}\right.
$$

and then to the gradient projection algorithm: given $\lambda^{0} \geq 0$ and $s^{0} \in L^{\infty}([0, T],\{0,1\})$ satisfying the constraint $\left\|s^{0}\right\|_{L^{1}([0, T])}=L T$, compute iteratively the sequence $\left(s^{k}, \lambda^{k}\right)_{(k>0)}$ :

$$
\left\{\begin{array}{l}
\lambda^{k+1}=P_{\mathbb{R}^{+}}\left(\lambda^{k}-\eta\left(\lambda^{k}-\int_{0}^{T} s^{k}(t) p^{k}\left(y_{0}, t\right) \mathrm{d} t\right)\right), \\
s^{k+1}(t)=P_{[0,1]}\left(s^{k}(t)-\eta\left(\epsilon_{1}^{-1}\left(1-2 s^{k}(t)\right)-\lambda^{k} p^{k}\left(y_{0}, t\right)+\mu^{k}\right)\right),
\end{array}\right.
$$

with $\eta>0$ small enough and $\mu^{k} \in \mathbb{R}$, the multiplier (determined by a line search algorithm) so that $\left\|s^{k+1}\right\|_{L^{1}(0, T)}=L T$. In (5.12), $p^{k}$ is the solution of the adjoint problem (4.7) associated to the control function $q^{k}=\lambda^{k} s^{k}$.

With the data and discretization used in Section 5.3, Table 3 provides some characteristics of the bang-bang controls with respect to the parameter $L \in[0,1]$. We use the numerical values $\left(\epsilon, \epsilon_{1}\right)=\left(10^{-6}, 10^{-4}\right)$. The 


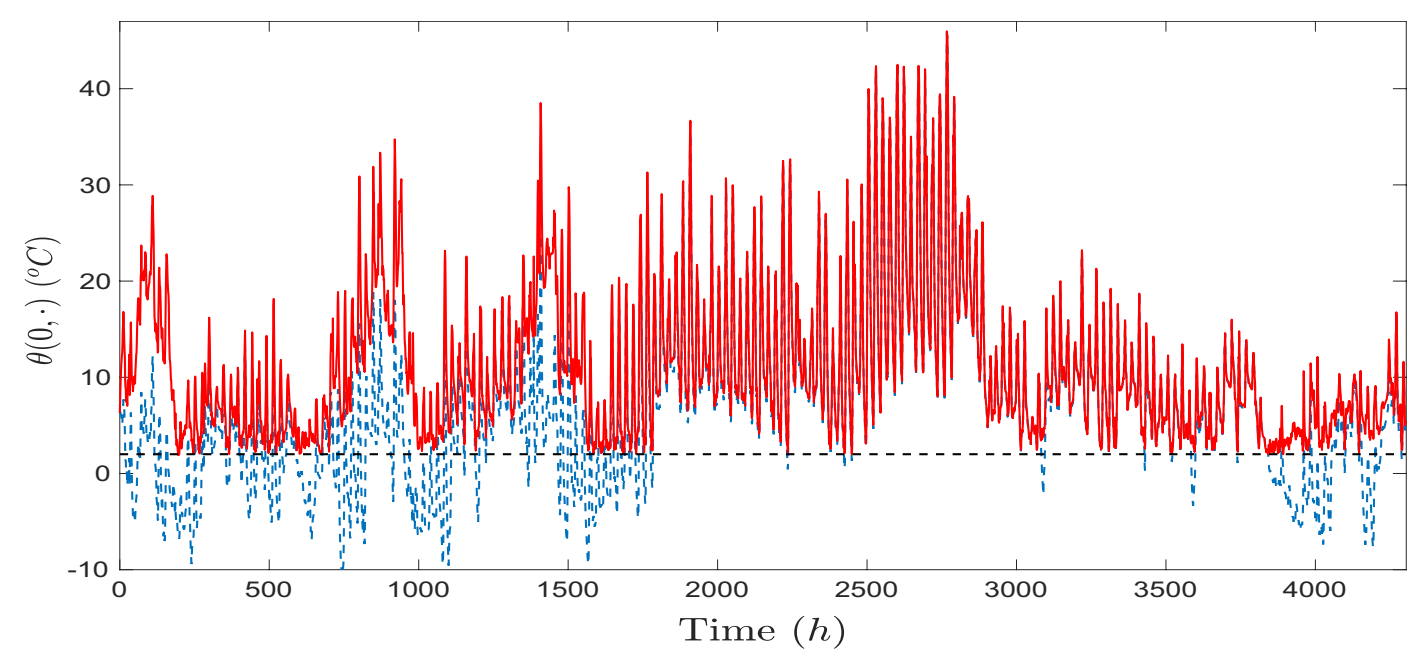

Figure 11 . Temperature $\theta(0, \cdot)$ at the road surface on $[0, T]$ in the controlled (red full line) and uncontrolled case (dot blue line).

TABLE 4. Characteristics of the temperature $\theta$ associated to the source (5.13).

\begin{tabular}{lllll}
\hline \hline$\delta$ & 0 & 50 & 54 & 55 \\
\hline$\|q\|_{L^{1}(0, T)}$ & $4.01 \times 10^{8}$ & $7.2 \times 10^{8}$ & $7.52 \times 10^{8}$ & $7.60 \times 10^{8}$ \\
$\|q\|_{L^{\infty}(0, T)}$ & $2.72 \times 10^{2}$ & $3.22 \times 10^{2}$ & $3.26 \times 10^{2}$ & $3.27 \times 10^{2}$ \\
$\left\|(\theta(0, \cdot)-\underline{\theta})^{-}\right\|_{L^{2}(0, T)}$ & $5.49 \times 10^{2}$ & $1.29 \times 10^{1}$ & 1.71 & 0. \\
$\left\|(\theta(0, \cdot)-\underline{\theta})^{-}\right\|_{L^{\infty}(0, T)}$ & 1.91 & $1.74 \times 10^{-1}$ & $2.92 \times 10^{-2}$ & 0. \\
\hline
\end{tabular}

projection algorithm is initialized with $\lambda^{0}=0$ and $s^{0}=L$. The variation of the bound $\lambda$ with respect to $L$ is small. Beyond $L=1 / 4$, the optimal $\lambda$ is almost constant. Actually, the amplitude of the control is driven by the data at the beginning of the time interval (corresponding to the colder period) where a large enough control is required, independently of the value of $L$. Figure 10 depicts the optimal control corresponding to $L=1 / 4$ while Figure 11 depicts the associated temperature $\theta(0, \cdot)$ at the road surface. Lower values of $L$ lead to larger amplitudes $\lambda$ and to an increasing number of switching points. We observe $L^{1}$-norm values of the same order as in the previous section $\left(e . g .\|q\|_{L^{1}(0, T)} \approx 4.57 \times 10^{8}\right.$ for $\left.L=1 / 10\right)$. Actually, for small values of $L$, we observe that the structure of the bang-bang control is similar to the optimal $L^{1}$ norm control (see Fig. 6 for small $\alpha$, e.g. $\alpha=10^{-7}$ ). The extreme value $L=1$ (for which the control is active on the whole time period) leads to a value $\lambda=2.34 \times 10^{2}$ : this value is in agreement with the result of the previous section which indicates that the optimal $L^{1}$ control satisfying the additional bound $\|q\|_{L^{\infty}} \leq 200$ does not allow to maintain the temperature greater than $\underline{\theta}$.

\subsection{Command law}

A priori, the optimal control we obtain in the previous section is nonlocal in the sense that its value $q(t)$ at the time $t \in(0, T)$ depends on the values of the data functions $f_{1}, f_{2}, \varepsilon$ on the full time interval. This may not be realistic from a practical point of view. A first way to circumvent, in the spirit of [4] is to split the time interval into a finite number of sub-intervals and then to compute optimal control on each subinterval.

A second way is to consider a source $q$ which depends explicitly on the data. According to (4.3), for $y_{0}=0$, if the source $q$ satisfies the condition $q+f_{1}(t)-f_{2}(t) \underline{\theta}-\sigma \varepsilon(t) \underline{\theta}^{4} \geq 0$, then the corresponding variable $\theta_{q}$ satisfies 

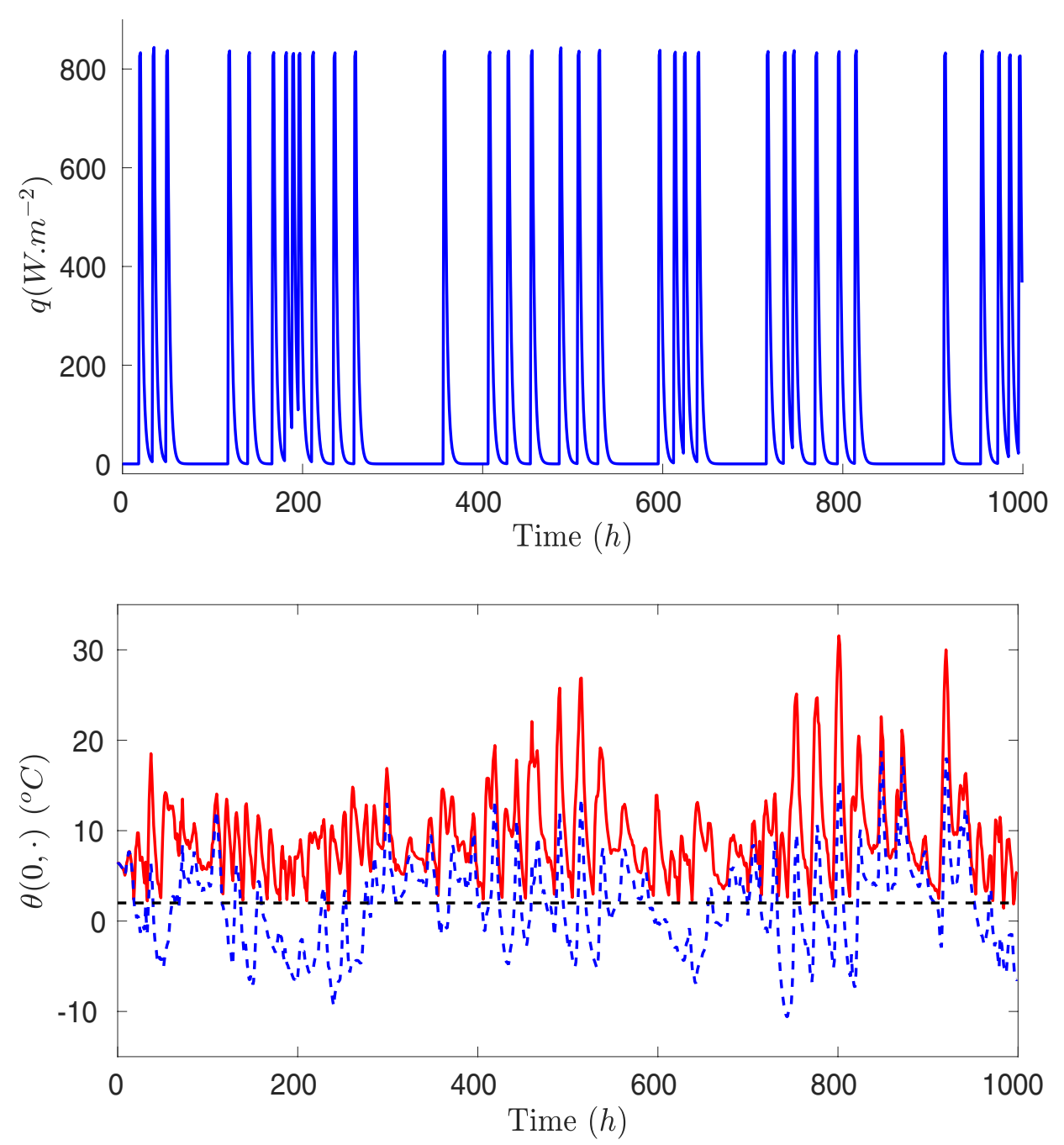

FiguRE 12. Source $q$ defined by (5.14) for $t \in[0,1000]$ (Top) and corresponding temperature $\theta(0, \cdot)$ at the road surface.

$\theta_{q}(0, t)-\underline{\theta} \geq 0$ for all $t \in(0, T)$. This suggests to consider, in our context where $y_{0}$ is close to zero, the following explicit source

$$
q(t)=\max \left(0,-\left(f_{1}(t)-f_{2}(t) \underline{\theta}-\sigma \varepsilon(t) \underline{\theta}^{4}\right)+\delta\right)
$$

for some real $\delta \geq 0$ large enough, dependent of $y_{0}$. Table 4 gives the $L^{1}$-norm of $q$ and the corresponding value of $\min \left((\theta(0, \cdot)-\underline{\theta})^{-}\right)$for some values of $\delta$. The value $\delta=55$ is large enough to satisfy the condition $\theta(0, \cdot) \geq 2^{\circ} C$ at the road surface. The corresponding $L^{1}$-norm $\|q\|_{L^{1}(0, T)} \approx 7.52 \times 10^{8}$ is of the same order as in the previous section.

Nevertheless, we observe that source of the form (5.13) is active on some period where the value of $\theta(0, \cdot)$ is (significantly) above $\underline{\theta}$. This is due to the large variations of the functions $f_{1}$ and $f_{2}$. A third way is therefore 
to consider source term $q$ which depends explicitly on the variable $\theta_{q}$, for instance as follows:

$$
q(t)=\left\{\begin{array}{cl}
0 & \text { if } \\
0 & \text { if } \quad \underline{\theta} \leq \theta(0, t-\delta) \leq \theta_{m} \quad \text { and } \quad \theta^{\prime}(0, t-\delta)>0, \\
f(t, \theta)\left(\theta(0, t-\delta)-\theta_{m}\right)^{-} & \text {else }
\end{array}\right.
$$

for some reals $\theta_{m}>\underline{\theta}, \delta \in(0, T)$ and a negative function $f$ which depends only at time $t$ on the temperature $\theta(s), s \in(0, t)$. Such source is active at time $t \geq \delta$ if and only if two situations occur: either when the value of $\theta(0, t-\delta)$ is below $\underline{\theta}$, either when the value of $\theta(0, t-\delta)$ is slightly above $\underline{\theta}$ (precisely, in the range $\left[\underline{\theta}, \theta_{m}\right]$ ) but decreases with respect to the time variable. Figure 12 depicts the source (5.14) associated to $\theta_{m}=273.15+3$, $\delta=1 \mathrm{~h}$ and to the corresponding temperature $\theta(0, \cdot)$. We take here simply for $f$ a large enough constant so that $\left\|(\theta(0, \cdot)-\underline{\theta})^{-}\right\|_{L^{\infty}(0, T)} \approx 0.08$ leading to $\|q\|_{L^{1}(0, T)} \approx 1.01 \times 10^{9}$ and $\|q\|_{L^{\infty}(0, T)} \approx 8.48 \times 10^{2}$. Although larger than for the optimal controls computed earlier, these values, associated to the source $q$ given by (5.14) explicitly in term of the history of the temperature, lead to promising results.

\section{Perspectives}

\subsection{Control by a road surface heating source: a different mathematical approach}

If the source $q$ is located at the road surface (i.e. $y_{0}=0$ ), we can construct a control by introducing a Signorini boundary condition as follows. For $y_{0}=0$, the right-hand side term of the first equation of (2.4) vanishes and the boundary condition at $y=0$ becomes:

$$
-k(0) \theta_{y}(0, t)=f_{1}(t)-f_{2}(t) \theta(0, t)-\sigma \varepsilon(t) \theta^{4}(0, t)+q(t), t \in(0, T) .
$$

Denoting $f(t, u)=-f_{1}(t)+f_{2}(t) u-\sigma \varepsilon(t) u^{4}$, we can eliminate $q$ and replace (6.1) by the Signorini type conditions:

$$
f(t, \theta(0, t))-\theta_{y}(0, t) \geq 0, \quad \theta(0, t)-\underline{\theta} \geq 0, \quad\left(f(t, \theta(0, t))-\theta_{y}(0, t)\right)(\theta(0, t)-\underline{\theta})=0,
$$

which are equivalent to $\theta_{y}(0, t)-f(t, \theta(0, t)) \in \gamma(\theta(0, t))$ where $\gamma: \mathbb{R} \rightarrow \mathcal{P}(\mathbb{R})$ is the following multivalued and maximal monotone operator:

$$
\gamma(z)=\emptyset \text { if } z<\underline{\theta}, \quad \gamma(z) \in]-\infty, 0] \text { if } z=\underline{\theta}, \quad \gamma(z)=\{0\} \text { if } z>\underline{\theta} .
$$

The formal variational problem under conditions (6.2) is: find $\theta$ with $\theta(0, \cdot) \geq \underline{\theta}$ such that for all $v \in H^{1}\left(0, h_{e}\right)$,

$$
\int_{0}^{h_{e}}\left(c(y) \theta_{t}(y, t) v(y)+k(y) \theta_{y}(y, t) v_{y}(y)\right) \mathrm{d} y+f(t, \theta(0, t)) v(0)+\gamma(\theta(0, t)) v(0) \ni 0,
$$

which is an extension of (3.2) with a multivalued and monotone term $\gamma(\theta)$ added to $\theta^{3}|\theta|$.

Results of Section 3 may be extended to this variational inclusion as done in [9], leading to an existence and uniqueness result for (6.3), and then to a control $q=-k(0) \theta_{y}(0, t)-f_{1}(t)+f_{2}(t) \theta(0, t)+\sigma \varepsilon(t) \theta^{4}(0, t)$. From a mathematical viewpoint, it would be interesting to study the link between this control of Signorini type and the $L^{1}$-optimal control exhibited in Section 4 .

\subsection{Optimal heating for the $2 \mathrm{D}$ diffusion-convection model}

We are interested in the 2D model described in (2.1)-(2.3) and in Figure 3. In this context, the energy lost by the fluid is expressed as follows:

$$
J_{2}\left(\theta_{f}\right)=v e_{2} C_{f} \int_{0}^{T} \int_{e_{1}}^{e_{1}+e_{2}}\left(\theta_{f}(t)-\theta_{\theta_{f}}(L, y, t)\right) \mathrm{d} y \mathrm{~d} t
$$


and then the optimal control problem is:

$$
\left\{\begin{array}{l}
\inf _{\theta_{f} \in L^{1}(0, T)} J_{2}\left(\theta_{f}\right), \text { subjected to: } \\
\theta_{f}(t) \geq 0, \theta_{\theta_{f}}(x, 0, t) \geq \underline{\theta}, \forall(x, t) \in(0, L) \times(0, T), \theta_{\theta_{f}} \text { solves }(2.1)-(2.3) .
\end{array}\right.
$$

For wider applications, it will be interesting to address this problem for a $3 \mathrm{D}$ model, allowing to take into account a longitudinal slant of the road.

\subsection{Storage problem}

Once the minimal energy has been estimated, there is the question of its origin. Indeed, in a context of energy transition, we must seek a renewable energy to heat the road. In an urban site, one can imagine using for example a heat network. In the case of an isolated road, one can seek to produce energy during the summer period, store it, and use it during winter. In this case, it is necessary to design a heat storage in an optimal way. Let us denote by $C$ the thermal capacity of the storage and denote by $\theta_{s}$ its temperature. Considering the thermal losses of the storage and taking energy in this storage, we can express the time evolution of $\theta_{s}$ as follows:

$$
C \frac{\mathrm{d} \theta_{s}}{\mathrm{~d} t}(t)=-A(t) \lambda_{s}\left(\theta_{s}(t)-\theta\left(y_{0}, t\right)\right)-p\left(t, \theta_{s}(t)\right)
$$

where $\lambda_{s}$ is the heat exchange coefficient between the storage (at temperature $\theta_{s}$ ) and the road (at temperature $\left.\theta\left(y_{0}, \cdot\right)\right), A$ is a $0-1$ function modeling the heat exchange activation and $p$ denotes a law (assumed known) of thermal losses of the storage. In the one dimensional case, we can then consider the following system:

$$
\left\{\begin{array}{lc}
c(y) \frac{\partial \theta}{\partial t}(y, t)-\frac{\partial}{\partial y}\left(k(y) \frac{\partial \theta}{\partial y}(y, t)\right)=\left(q(t)+A(t) \lambda_{s}\left(\theta_{s}(t)-\theta\left(y_{0}, t\right)\right)\right) \delta\left(y_{0}\right), & (y, t) \in Q_{T}, \\
-k(0) \frac{\partial \theta}{\partial y}(0, t)=f_{1}(t)-f_{2}(t) \theta(0, t)-\sigma \varepsilon(t) \theta^{4}(0, t), \frac{\partial \theta}{\partial y}\left(h_{e}, t\right)=0, & t \in(0, T), \\
\theta(y, 0)=\theta_{0}(y), & y \in\left(0, h_{e}\right), \\
C \frac{d \theta_{s}}{d t}(t)=-A(t) \lambda_{s}\left(\theta_{s}(t)-\theta\left(y_{0}, t\right)\right)-p\left(t, \theta_{s}(t)\right), & t \in(0, T) .
\end{array}\right.
$$

The optimization of the capacity and the source $q$ then leads to the following optimization problem:

$$
\begin{cases}\inf _{C, A, q}\left(\epsilon_{1} C+\left(1-\epsilon_{1}\right) \int_{0}^{T} q(t) \mathrm{d} t\right) & \text { subjected to: } \\ C>0, A(t) \in\{0,1\}, q(t) \geq 0, \theta_{C, A, q}(0, t) \geq \underline{\theta}, & \forall t \in(0, T) \\ \left|\theta_{s}(T)-\theta_{s}(0)\right| \leq d, & \theta_{C, A, q} \text { solves }(6.7)\end{cases}
$$

where $\epsilon_{1}>0$ and where $d \geq 0$ is a given (small) real allowing to maintain the storage at its initial temperature after one year $(T)$. This storage problem could be adapted as well to the $2 \mathrm{D}$ dimensional problem of Section 6.2 .

\section{Conclusion}

We have presented in this work an original approach to evaluate the minimal energy for heating a road in order to keep its surface frost free. With the help of a transient one dimensional thermal road model, we look for a heat source inserted in the pavement that allows to maintain the surface temperature above a threshold value. Theoretical results are established for the uniqueness and existence of a solution to the direct problem and to the optimal control problem. For these results, we take into account the nonlinear term due to the Stefan-Boltzmann law for which an emissivity can depend on time. We analyze the different controls obtained numerically according to the $L^{1}, L^{2}$ and $H^{1}$ norm. The $L^{1}$-norm has the most relevant 
physical meaning since it corresponds to the energy for heating the road and that we want to minimize. On the other hand, the control is sparse and leads to $L^{\infty}$-norm higher than controls corresponding to the $L^{2}$ and $H^{1}$ minimization. Furthermore, for a better application, we study a bang-bang control taking only two values and allowing then to bound the control (the heat power). Some estimates of the optimal energy are given in Section 5: the total energy needed to keep the road surface temperature over $2^{\circ} \mathrm{C}$ during a winter with snow is about $5.10^{8} \mathrm{~J} \simeq 139 \mathrm{kWh} \mathrm{m}^{2}$ of road, with minimal and maximal values per $\mathrm{m}^{2}$ respectively equal to $124 \mathrm{kWh}$ and $213 \mathrm{kWh}$. Moreover, the $L^{\infty}$-norm of the optimal power $q$ ranges in $240-500 \mathrm{~W} \mathrm{~m}^{-2}$. In [15], some experiments of heating roads by electric heating cables show needed power and energy equal respectively to $500-750 \mathrm{~W} \mathrm{~m}^{-2}$ and $100-170 \mathrm{kWh} \mathrm{m}^{-2}$. In $[7,10]$ needed power and energy are experimentally evaluated around $400-500 \mathrm{~W} \mathrm{~m}^{-2}$ and $130-350 \mathrm{kWh} \mathrm{m}^{-2}$ for de-icing obtained by the circulation of a coolant in pipes inserted in the road. Although the meteorology data are not the same with respect to the above mentioned studies, one notices that the power and energy provided by our simulations have the same order of magnitude than those obtained experimentally for systems based on electric heating or coolant circulation in pipes. As mentioned in Section 6, we plan to extend our works to the heating system by circulation of a coolant in a bonding porous layer of the road. We will be able to compare our numerical results with experimental measurements collected on the Egletons demonstrator described in Section 1. Following [13], it is also interesting to analyze in our nonlinear setting, the influence on the optimal controls of the perturbation of the data $f_{1}, f_{2}$ and $\varepsilon$, due notably to measurements.

\section{REFERENCES}

[1] F. Ammar-Khodja, S. Micu and A. Münch, Controllability of a string submitted to unilateral constraint. Ann. Inst. Henri Poincaré Anal. Non Linéaire 27 (2010) 1097-1119.

[2] S. Asfour, F. Bernardin and E. Toussaint, Experimental validation of 2d hydrothermal modelling of porous pavement for heating and solar energy retrieving applications. To appear in: Road Mater. Pavement Des. J. doi: 10.1080/14680629.2018.1525418 (2018).

[3] S. Asfour, F. Bernardin, E. Toussaint and J.-M. Piau, Hydrothermal modeling of porous pavement for its surface de-freezing. Appl. Therm. Eng. 107 (2016) 493-500.

[4] B. Azmi and K. Kunisch, Receding horizon control for the stabilization of the wave equation. Discrete Contin. Dyn. Syst. 38 (2018) 449-484.

[5] L. Bouilloud and E. Martin, A coupled model to simulate snow behavior on roads. J. Appl. Meteorol. Climatol. 45 (2006) $500-516$.

[6] E. Casas, C. Clason and K. Kunisch, Parabolic control problems in measure spaces with sparse solutions. SIAM J. Control Optim. 51 (2013) 28-63.

[7] M. Chen, S. Wu, H. Wang and J. Zhang, Study of ice and snow melting process on conductive asphalt solar collector. Solar Energy Mater. Solar Cells 95 (2011) 3241-3250.

[8] L.-P. Crevier and Y. Delage, Metro: a new model for road-condition forecasting in canada. J. Appl. Meteorol. 40 (2001) 2026-2037.

[9] G. Duvaut and J.-L. Lions, Inequalities in mechanics and physics. Translated from the French by C.W. John, Grundlehren der Mathematischen Wissenschaften 219. Springer-Verlag, Berlin-New York (1976).

[10] W. Eugster, Road and bridge heating using geothermal energy. overview and examples. In: Proceedings European Geothermal Congress 2007 Unterhaching (2007).

[11] R. Mallick, B. Chen, S. Bhowmick and M. Huken, Capturing solar energy from asphalt pavements. In: International ISAP Symposium on Asphalt Pavements and Environment. Zurich, Switzerland (2008).

[12] A. Mammeri, L. Ulmet, C. Petit and A. Mokhtari, Temperature modelling in pavements: the effect of long- and short-wave radiation. Int. J. Pavement Eng. 16 (2015) 198-213.

[13] J. Martínez-Frutos, M. Kessler, A. Münch and F. Periago, Robust optimal Robin boundary control for the transient heat equation with random input data. Int. J. Numer. Methods Eng. 108 (2016) 116-135.

[14] X. min Zhou, Z.J. Yang, C. Chang and G. Song Numerical assessment of electric roadway deicing system utilizing emerging carbon nanofiber paper. J. Cold Regions Eng. 26 (2012) 1-15.

[15] K. Morita and M. Tago, Operational characteristics of the gaia snow-melting system in Ninohe, Iwate, Japan. In: World Geothermal Congress 2000. Beppu-Morioka, Japan (2000) 3511-3516.

[16] A. Münch and F. Periago, Numerical approximation of bang-bang controls for the heat equation: an optimal design approach. Syst. Control Lett. 62 (2013) 643-655.

[17] E. Sachs, A parabolic control problem with a boundary condition of the Stefan-Boltzmann type. Z. Angew. Math. Mech. 58 (1978) 443-449. 
[18] A. Scacht, M. Munk, C. Busen, M. Osser and B. Steinauer, Application of a porous interlayer for road temperature control. In: XIV International Winter Road Congress. Institute for Road and Traffic Engineering, RWTH Aachen university, Deutschland, PIARC, Andoraa la Vella (2014).

[19] T. Scharf, La route thermorégulée. In: ARTE Futuremag, Les routes de demain. Paris, France (2016).

[20] Z. Tang, Z. Li, J. Qian and K. Wang, Experimental study on deicing performance of carbon fiber reinforced conductive concrete. J. Mater. Sci. Technol. 21 (2005) 113-117.

[21] W. Yu, X. Yi, M. Guo and L. Chen, State of the art and practice of pavement anti-icing and de-icing techniques. Sci. Cold Arid Reg. 6 (2014) 14-21.

[22] J. Zhang, D. Das and R. Peterson, Selection of effective and efficient snow removal and ice control technologies for cold-region bridges. J. Civil Environ. Architectural Eng. 3 (2009) 1-14.

[23] H. Zhao, Z. Wu, S. Wang, J. Zheng and G. Che, Concrete pavement deicing with carbon fiber heating wires. Cold Regions Sci. Technol. 65 (2011) 413-420. 